\title{
Material stability analysis of rock joints
}

\author{
Jérôme Duriez, Félix Darve and Frédéric-Victor Donzé and François Nicot
}

\begin{abstract}
Résumé
In order to predict rockfalls, the failure of rock joints is studied. Considering these failures as constitutive instabilities, the second order work criterion is used, since it explains all divergence instabilities (flutter instabilites are excluded). The bifurcation domain and the loading directions of instabilities, which fulfil the criterion, are determined for any piecewise linear constitutive relation. The instability of rock joints appears to be ruled by coupling features of the behaviour (e.g., dilatancy). Depending on the loading parameters, instabilities can lead to failure, even before the plastic limit criterion. Results for two given constitutive relations illustrate the approach. Some given loading paths are especially considered. Constant volume (undrained) shear and $\tau$-constant paths are stable or not depending on the link between the deviatoric stress and strain along undrained paths, as found for soils. Some unstable loading paths are illustrated. Along these paths, failure before plastic limit criterion is possible. The corresponding failure rules are determined.
\end{abstract}

Rock slopes present different types of defects at all scale. We call rock joints the discontinuities (e.g., in mechanical properties) at macroscopic scale and we assume that rock joints have the greatest influence on the stabilities of rock slopes. This is why geomechanical stability analyses need to represent accurately rock joints' failures. We thus focus on the mechanical behaviour of such joints and, in the framework of a $2 \mathrm{D}$ assumption, only four scalar variables will be used to describe the corresponding mechanical state. Two stress components are considered : one normal, denoted $\sigma$ (considered positive in compression), and one tangential, denoted $\tau$. On the other hand, relative displacements occuring along the joint are considered, with a normal component $u$ (positive in compression) and a tangential component $\gamma$.

We define here, as in the general plasticity theory that failure is obtained when relative displacements along the joint (that we can call "deformation", from a general point of view) go on under a constant loading, by the existence of limit stress states. Failure of rock joint is obtained for example during constant normal load (CNL) shearings, defined by a constant value of $\sigma$, once $\tau$ reaches a peak or a plateau $[1,2,3,4]$ : under these conditions $\gamma$ increases continuously while stresses are constant. This situation in a rock slope would trigger rockfalls. Such analysis of failure introduces directly the concept of limit stress states, for which stresses do not vary anymore : $\overrightarrow{d \sigma}=(d \tau, d \sigma)=\overrightarrow{0}$, while relative displacements still evolve : $\overrightarrow{d l}=(d \gamma, d u) \neq \overrightarrow{0}$. 
As in the previous example for $\tau$ peak during CNL shearing, limit stress states occur first of all (but not exclusively) on the plastic limit criterion the frontier, in the stress space, of all admissible stress states, e.g. the MohrCoulomb criterion. For this reason failures of materials in general (not only rock joints) were first considered through limit analyses (by Drucker [5]), also denoted as limit equilibrium methods in the framework of geomechanics. The corresponding static limit theorem focuses on stress states corresponding to plastic limit criterion, generally within the assumption that such stress states are reached for the entire body. Such analyses were applied to rock joints and rock slopes stability by several authors $[6,7,8,9]$.

Alternatively, the use of a constitutive relation linking $\overrightarrow{d \sigma}$ to $\overrightarrow{d l}$ allows also to analyse these limit stress states. If a rate-independent behaviour is assumed for the rock joint, such constitutive relation can be expressed through a constitutive matrix $M_{h}$ that depends on the hardening parameter $h$, and on the loading direction $\vec{d}=\overrightarrow{d l} /\|\overrightarrow{d l}\|$. This matrix links $\overrightarrow{d \sigma}$ to $\overrightarrow{d l}$ (more details about these results can be found for example in [10]) :

$$
\overrightarrow{d \sigma}=M_{h}(\vec{d}) \overrightarrow{d l}
$$

We now introduce (after Darve [11]) tensorial zones, defined as parts of the $(d u, d \gamma)$ space in which matrix $M_{h}$ is constant for the corresponding set of vectors $\vec{d}$. Then, if a given tensorial zone is assumed, limit stress states are equivalent to cancellation of the determinant of matrix $M_{h}$ :

$$
\overrightarrow{d \sigma}=M_{h} \overrightarrow{d l}=\overrightarrow{0}, \text { with } \overrightarrow{d l} \neq \overrightarrow{0} \Leftrightarrow \operatorname{det}\left(M_{h}\right)=0
$$

Having described failure of a rock joint, stability of a rock slope can finally be assessed using such constitutive relation, since, with an appropriate numerical method, mechanical states of all rock joints can be determined precisely during a given loading history.

Compared to previous limit analyses, such stress-strain analyses are fundamentally different. Besides a plastic limit criterion, a constitutive relation is required, but sharper descriptions of failures can be obtained. On one hand, for example, with limit analyses the failure mechanisms (the directions of displacement fields at failure) can be determined, using - associated, generally - flow rules, only when corresponding stress states reach their yield surfaces (which are here equivalent to plastic limit criterion since no hardening is considered). On the other hand, with stress-strain analyses, the displacements are fully determined in any cases, and not only on failure. Finally, compared to limit analyses, the second approach presents several advantages. First, the mechanical history is completely determined, which has an influence for hardening behaviours. Second, the stress states can be computed, and do not require to be assumed. Thus critical rock joints with stress states obeying to plastic limit criterion can be determined in rock slopes. Finally, and we will discuss it later, failures of geomaterials can occur before the plastic limit criterion is reached, and such failures can thus not be explained by the limit analyses. 
A comparison between the two approaches was presented by Adhikary and Dyskin [9]. In their work, results of the limit analysis overestimated the failure level by at least $70 \%$, compared to experimental results, whereas results obtained by the stress-strain analysis approached the experimental ones with a maximal error of $15 \%$. For the authors, the differences were caused by the assumption of an homogeneous stress field (which obeys the plastic limit criterion everywhere) in the limit analysis and not in the stress-strain analysis.

The framework of this work is thus the assessment of rock slope stability using stress-strain analysis. To do so, we will analyse in this paper the rock joints failure, using corresponding constitutive matrices, and focusing on the limit stress states evocated before. For nonassociated materials (like rock joints $[12,13])$, we also have to take into account the generalized limit states that can occur before the plastic limit criterion. Contrary to classical limit stress states, these generalized limit states do not only involve the stress values (displacements also have to be taken into account), and they depend on the loading path. They were discussed for example by Darve et al. [14]. Such states can also lead to failures, thus before plastic limit criterion, depending on the loading parameters. Undrained triaxial tests on loose sands provide a typical example. During such tests, peaks of $q^{1}$ are classicaly observed before Mohr-Coulomb plastic limit condition, and corresponding failures can develop if the test is stress-controlled, rather than strain-controlled [15]. Another example can be observed along $q$ constant loading paths. The generalized limit state corresponds in this case to the maximum of volumic strain that can be measured before plastic limit criterion, and failures are observed after a volumetric perturbation (e.g., after an injection of a small amount of water) $[16,17]$.

We shall mention a second type of failures that can be encountered before the plastic limit criterion is reached, when increasing plastic deformation localizes in shear or compaction/dilation bands $[18,19,20]$. Such failures could be explained by Rice's criterion [21,22], but they differ from the previous examples because of their localized pattern, which did not appear in previous examples. Indeed, the first kind of failures, before plastic limit criterion, that we mention, are refered to as diffuse [23].

In such cases, a new tool, different from the plastic limit criterion or Rice's criterion, is required to explain the failures. To reach this goal, Darve et al. $[24,25,26]$ reinterpreted these failures through the notions of bifurcation and instablitity (as Rudnicki and Rice [21] did). In general any failure can be seen as a bifurcation, since failure corresponds to a discontinuous transition from a quasi-static regime of deformation, to a dynamic one, under continuous loading. Regarding instability, the definition of Lyapunov [27] (see for example [14]) is considered. In this sense, and roughly speaking, an unstable state is reached when "small" loadings can result in "large" responses. It is the case for failures at limit stress states : once the limit state reached, any additional infinitesimal

1. During such tests, two of the three principal stresses are equal, to $\sigma_{\text {lat }}$. Denoting the third principal stress $\sigma_{a x}$, it is defined as $q=\sigma_{a x}-\sigma_{l a t}$. 
load will imply failure, and large deformations.

Within this framework, Hill's necessary criterion of instability [28] gave the starting point to define the second order work criterion [29] which explained successfully the diffuse failures presented before [14, 24, 30]. First theoretical explanations obtained by this approach have been confirmed experimentally $[31,32]$. The great advantage of the second order work criterion is its ability to explain all kinds of failures by divergence instabilities : sudden monotonous and unbounded increases of the strains, starting from some initial state, denoted as unstable. Failures on plastic limit criterion, we first evocated, are indeed also explained as a particular case in the framework of the second order work criterion. Localized failures are also explained since Rice's criterion can be seen as a particular case according to linear algebra [33, 34]. Indeed failures always correspond to instabilities, whereas the reciprocal is false, as explained by Daouadji et al. [30] for example. And the second order work is the most general criterion explaining all divergence instabilities [35], excluding the flutter instabilities which correspond to suddenly cyclically increasing strains.

We will apply this criterion to rock joints constitutive relations to study rock joints failure through a constitutive point of view and we will compare it to previous results obtained for a soil body. Section 1 presents general results, valid for any incremental piecewise-linear rock joints constitutive relations. Section 2 focuses on two rock joint constitutive relations, proposed previously by Duriez et al. [13] : a piecewise-linear one, the other incrementally nonlinear. The corresponding results, as well as a parametric study are presented. In sections 3 and 4 the behaviour of rock joints along displacement and stress proportional loading paths will be considered. The purpose of such paths is to clearly illustrate what happens when conditions of the second order work criterion are fullfilled, and to compare to soil case, for which experimental evidences of negative second order work (and corresponding failures) were observed along such paths. In the final section 5 failure rules [14] will be derived, which characterize these failures occuring before plastic limit criterion, as flow rules characterize the failures corresponding to plastic limit criterion.

\section{Second order work criterion and joint stability analysis}

For 3D media, at a material point scale, infinitesimal variations of the mechanical state can be described by two vectors belonging to $\mathbb{R}^{6}: \overrightarrow{d \sigma}$ and $\overrightarrow{d \epsilon}$,

derived from the two symmetric tensors $\boldsymbol{d} \boldsymbol{\sigma}$ and $\boldsymbol{d} \boldsymbol{\varepsilon}$. The second order work is defined as : (see for example the synthetizing paper by Daouadji et al. [30])

$$
d^{2} w=\overrightarrow{d \sigma} \cdot \overrightarrow{d \epsilon}={ }^{t} \overrightarrow{d \sigma} \overrightarrow{d \epsilon}
$$

For a part of a rock joint with an homogeneous mechanical state, and considering only one tangential and one normal direction, two vectors $\overrightarrow{d \sigma}$ and $\overrightarrow{d l}$ belonging 
to $\mathbb{R}^{2}$ are used, such that :

$$
d^{2} w=\overrightarrow{d \sigma} \cdot \overrightarrow{d l}={ }^{t} \overrightarrow{d \sigma} \overrightarrow{d l}
$$

Due to the existence of a constitutive matrix $M_{h}$ which depends on the loading direction $\vec{d}$, the sign of $d^{2} w$ depends only on direction $\vec{d}$ (for a given mechanical state) :

$$
d^{2} w=\overrightarrow{d l} \cdot \overrightarrow{d \sigma}=\overrightarrow{d l} \cdot M_{h}(\vec{d}) \overrightarrow{d l}=\|\overrightarrow{d l}\|^{2} \vec{d} \cdot M_{h}(\vec{d}) \vec{d}
$$

The second order work criterion focuses whether the sign of $d^{2} w$ is negative or not. The first analysis of R. Hill [28] has been recently complemented by establishing a link between negative values of second order work and bursts of kinetic energy at an unstable state leading to failure [36, 37, 38]. These bursts are a clear proof of an effective failure, validating the second order criterion as a proper failure criterion. Here, the considered definition of (un)stability is slightly different from the one of Lyapunov we stated previously. Nevertheless, links between the two exist, as it was discussed by Darve et al. [14].

Due to the directional feature of second order work's sign, it was defined : - directions of instability, also denoted unstable directions, as the loading directions which trigger $d^{2} w \leqslant 0$

- a bifurcation domain, as the set of mechanical states for which at least one direction of instability exists. Such states are denoted as unstable ones.

Failure occurs under the three following necessary and sufficient conditions : the stress state belongs to the bifurcation domain, the loading direction is an unstable one, and appropriate loading parameters are used [30]. As for the example of undrained triaxial test on loose sand (as mentioned in introduction) the loading parameters have to involve both stress and strain increments, so that failure occurs. Otherwise, the existing instability does not lead to failure.

Note that the first and second conditions are linked together since no direction of instability can be obtained if the stress state is not an unstable one, i.e. if it is outside the bifurcation domain.

An alternative approach would be to apply the notion of controllability proposed by Nova $[39,40]$ to our interface strains and stress variables. This would provide the possibility determine the mechanical states and loading directions for which the existence and uniqueness of the mechanical response may be lost (depending on the loading parameters) [41]. This corresponds to the three conditions of failure we stated previously. It has been demonstrated $[36,30]$ that the notion of controllability proposed by Nova and the application of the second order work criterion can be developed in a unique framework.

For the rest of section 1, we now consider any rock joint constitutive relation with a finite number of tensorial zones. Since constitutive matrix depends on loading directions $\vec{d}$, recall that tensorial zones are defined as parts of loading direction space (here the plane $(d \gamma, d u)$ ) in which this matrix is constant. For elastic models, one tensorial zone is assumed, whereas an infinity can be obtained 
with incrementally nonlinear (INL) formalisms [42, 43] (applied to rock joints in $[10,13])$.

General results about second order work are presented in this framework.

\subsection{Bifurcation domain and directions of instability}

If the direction of $\overrightarrow{d l}=(d u, d \gamma)$ belongs to the set of loading directions defining a given tensorial zone, it is here possible to write :

$$
\left(\begin{array}{c}
d \tau \\
d \sigma
\end{array}\right)=\left(\begin{array}{ll}
G_{\gamma} & G_{u} \\
N_{\gamma} & N_{u}
\end{array}\right)\left(\begin{array}{c}
d \gamma \\
d u
\end{array}\right)=M\left(\begin{array}{c}
d \gamma \\
d u
\end{array}\right)
$$

In this case :

$$
d^{2} w=d \sigma d u+d \tau d \gamma=N_{u} d u^{2}+\left(G_{u}+N_{\gamma}\right) d u d \gamma+G_{\gamma} d \gamma^{2}
$$

If $d \gamma=0$, which is the case for œedometric compressions, we get $d^{2} w=$ $N_{u} d u^{2}$. Since $d u \neq 0$ (otherwise $\overrightarrow{d l}=\overrightarrow{0}$ which is not to be considered), we have in this case $d^{2} w \leqslant 0 \Leftrightarrow N_{u} \leqslant 0$. Moduli $N_{u}$ being a normal stiffness, this may occur in high porosity, compacting rocks showing compaction bands [44]. This corresponds to instabilities leading to localized failures $[45,46]$. However, according to Mollema and Antonellini [44], restricting to rocks with porosity lower than $20 \%$ dismisses this special case; as an example, porosity for granite and gneiss is around few percent. In this case no instability can occur during an œedometric compression, as it could be figured out.

In order to find out conditions leading to $d^{2} w \leqslant 0$ we can now consider $d \gamma \neq 0$. Then :

$$
d^{2} w=d \gamma^{2}\left(N_{u}\left(\frac{d u}{d \gamma}\right)^{2}+\left(G_{u}+N_{\gamma}\right) \frac{d u}{d \gamma}+G_{\gamma}\right)
$$

Sign of $d^{2} w$ is hence the same as the one of second order polynom $P$, defined by :

$$
P(X)=N_{u} X^{2}+\left(G_{u}+N_{\gamma}\right) X+G_{\gamma}
$$

We still consider $N_{u}>0$, thus two conditions have to be fullfilled to get $d^{2} w \leqslant 0$. The first condition concerns the discriminant which must be positive :

$$
\Delta=\left(G_{u}+N_{\gamma}\right)^{2}-4 N_{u} G_{\gamma} \geqslant 0 \Leftrightarrow\left(G_{u}+N_{\gamma}\right)^{2} \geqslant 4 N_{u} G_{\gamma}
$$

This condition is the equation of the bifurcation domain, which gathers all mechanical states obeying to Equation (10). It can be physically interpreted as follow. Let consider $N_{u}$ and $G_{\gamma}$ as diagonal rigidities, because they link together normal variables ( $d \sigma$ with $d u$ ) or tangential variables $(d \tau$ with $d \gamma$ ), and hence $G_{u}$ and $N_{\gamma}$ as nondiagonal ones. Equation (10) expresses that instability can be obtained once the magnitude of nondiagonal rigidities becomes greater than the one of the diagonal ones. Classical definition of failure, through limit plasticity and cancellation of determinant of constitutive matrices, relies also on 
such comparison between diagonal and nondiagonal rigidities, as explained in a previous work [13].

Once in the bifurcation domain, the second condition concerns direction of $\overrightarrow{d l}$, i.e. ratio $d u / d \gamma$. It must belong to the set delimited by the two roots which now exist :

$$
d u / d \gamma \in S=\left[\frac{-\left(G_{u}+N_{\gamma}\right)-\sqrt{\Delta}}{2 N_{u}} ; \frac{-\left(G_{u}+N_{\gamma}\right)+\sqrt{\Delta}}{2 N_{u}}\right]
$$

This constitutes the set of directions of instability which exist once in the bifurcation domain. $S$ being an interval, the set of corresponding $\overrightarrow{d l}$ forms a cone in $(d u, d \gamma)$ plane. Two branches of the cone correspond to directions leading to an exact nil value of $d^{2} w$, whereas directions strictly inside the cone lead to strictly negative values of $d^{2} w$. For constitutive relations with more than one tensorial zone, it must be verified if directions of instability of set $S$ belong to the expected tensorial zone.

Through the constitutive matrix, interval $S$ of unstable displacement directions becomes also a stress direction interval, gathering the unstable stress directions. These unstable stress directions form also a cone in Mohr plane, as mentioned in other studies, for soil case [47, 14, 48]. Note that in $[14,48]$ more than one unstable cone could be found depending on the considered mechanical state. This would also be the case for piecewise linear relations, if Equation (10) is fullfilled for different matrices corresponding to different tensorial zones (and if corresponding directions of instability belong to the right tensorial zone).

\subsection{Orientation of the instability cones}

Unstable stress directions cones found previously for soils [47, 14, 48] point generally towards stress origin (the second type of cones of $[14,48]$ excepted). To determine what happens for unstable stress cones for rock joints, we consider $d \sigma_{k}{ }^{u}, k \in\{1 ; 2\}$, the components of the branchs of the unstable stress cone in Mohr plane, see Figure 1. After calculations detailled in appendix, we deduce

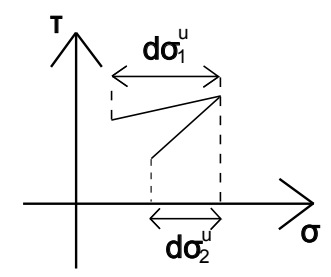

Figure 1 - Definition of $d \sigma_{k}{ }^{u}$ for the cones of instability in Mohr plane

links between signs of $d \sigma_{k}{ }^{u}$ and $d \gamma$, depending on the comparison between the coupling moduli $N_{\gamma}$ and $G_{u}$, see Table 1 .

Table 1 is mathematically valid as soon as the stiffness $N_{u}$ and determinant $\operatorname{det}(M)$ are positive. The orientation of instability cones depends on the coupling 


\begin{tabular}{c|c|c} 
& $d \gamma<0$ & $d \gamma>0$ \\
\hline$G_{u}>N_{\gamma}$ & $\begin{array}{l}\text { Unstable stress cone } \\
\text { in half space } d \sigma>0\end{array}$ & $\begin{array}{l}\text { Unstable stress cone } \\
\text { in half space } d \sigma<0\end{array}$ \\
$G_{u}<N_{\gamma}$ & $\begin{array}{l}\text { Unstable stress cone } \\
\text { in half space } d \sigma<0\end{array}$ & $\begin{array}{l}\text { Unstable stress cone } \\
\text { in half space } d \sigma>0\end{array}$
\end{tabular}

TABLE 1 - Orientation in Mohr plane of the cones of instability when they exist

moduli of the rock joints behaviour : $N_{\gamma}$ related to contractancy-dilatancy, and $G_{u}$ related to influence of compressions over $\tau$. They point, for example in $d \sigma<0$ direction in two cases : if $G_{u}>N_{\gamma}$ and $d \gamma>0$, or if $G_{u}<N_{\gamma}$ and $d \gamma<0$.

\subsection{Associativeness versus nonassociativeness}

The second order work $d^{2} w$ corresponds to the quadratic form of the symmetric part, $M^{S}$, of the constitutive matrix $M$, since

$$
d^{2} w=\overrightarrow{d l} .(M \overrightarrow{d l})=\overrightarrow{d l} .\left(M^{S} \overrightarrow{d l}\right)
$$

Through linear algebra a direct link between the sign of $d^{2} w$ and the one of $\operatorname{det}\left(M^{S}\right)$ appears (see also previous discussions by Nicot et al. $[49,50]$ ). This leads to two consequences.

First, the bifurcation domain can be determined focusing on $\operatorname{det}\left(M^{S}\right)$. From Equation (12), we conclude that some $\overrightarrow{d l}$ directions of instability exist if and only if $\operatorname{det}\left(M^{S}\right) \leqslant 0$. This means that $\operatorname{det}\left(M^{S}\right) \leqslant 0$ is the equation of the bifurcation domain. This corresponds to previous Equation (10), since we have :

$$
M^{S}=\left(\begin{array}{cc}
G_{\gamma} & \frac{G_{u}+N_{\gamma}}{2} \\
\frac{G_{u}+N_{\gamma}}{2} & N_{u}
\end{array}\right)
$$

Then :

$$
\operatorname{det}\left(M^{S}\right)=G_{\gamma} N_{u}-\left(\frac{G_{u}+N_{\gamma}}{2}\right)^{2}=-\frac{\left(G_{u}+N_{\gamma}\right)^{2}-4 G_{\gamma} N_{u}}{4}
$$

According to Equation (10)

$$
\operatorname{det}\left(M^{S}\right)=-\frac{\Delta}{4}
$$

The two conditions : $\Delta \geqslant 0$ and $\operatorname{det}\left(M^{S}\right) \leqslant 0$ are equivalent. Since stress states are limited by the plastic limit criterion $: \operatorname{det}(M)=0$, the bifurcation domain is contained between $\operatorname{det}\left(M^{S}\right)=0$ and $\operatorname{det}(M)=0$. This allows to distinguish associative cases, from nonassociative ones. As soils, associative rock joints present 
a symmetric constitutive matrix (see [13] for example) $: M=M^{S}$. In this case, the both conditions $\operatorname{det}\left(M^{S}\right)=0$ and $\operatorname{det}(M)=0$ are fulfilled simultaneously. This leads to the impossibility of failure before plastic limit criterion for associative rock joints, as it was proposed for soils by Darve \& Laouafa [51].

Then, if a given mechanical state is now assumed, $\operatorname{det}\left(M^{S}\right)$ depends on the loading direction, since it is the case of constitutive matrix $M$. Hence, the link between directions of instability (leading to $d^{2} w \leqslant 0$ ) and directions leading to $\operatorname{det}\left(M^{S}\right) \leqslant 0$ can also be studied.

As explained in section 1.1, directions of instability correspond to Equation (11), which requires that the bifurcation domain is reached : $\Delta \geqslant 0$. Equivalence $\Delta \geqslant 0 \Leftrightarrow \operatorname{det}\left(M^{S}\right) \leqslant 0$ just being proved, this means that the directions of instability lead to $\operatorname{det}\left(M^{S}\right) \leqslant 0$ : the set of directions of instability is included inside the set of directions leading to $\operatorname{det}\left(M^{S}\right) \leqslant 0$. This result was presented for soils by Darve \& Laouafa [51], and it is now also valid for rock joints. Nevertheless, the reciprocal is false. Indeed, directions leading to $\operatorname{det}\left(M^{S}\right) \leqslant 0 \Leftrightarrow \Delta \geqslant 0$ do not belong necessarily to the interval of directions of instabilitity of Equation (11). This will be illustrated in section 2.2.3.

\section{Application to Quadri and INL2 constitutive relations}

Focus is now laid on two particular rock joint constitutive relations. They are first presented, before studied in the framework of the second order work criterion.

\subsection{The Quadri and INL2 relations}

Constitutive relations can be distinguished depending on the number of tensorial zones they provide. Relations with only one tensorial zone describe only elastic behaviours. A greater number of tensorial zones allows to describe more efficiently the mechanical behaviour of geomaterials. We will then use the $Q u a$ dri and INL2 constitutive relations presented in a previous work [13] because they have four and an infinity of tensorial zones respectively. They are both of interpolation type, and partly defined outside classical elastoplastic formalism : they do not rely on a decomposition of strain between elastic and plastic parts. Their expressions rely on two calibration paths. First, a constant normal displacement (CND) path, with $(d u=0, d \gamma=c s t)$ is considered. It provides four moduli, corresponding to the slopes of the resulting curves $\tau(\gamma)$ and $\sigma(\gamma)$ in loading $(d \gamma>0)$, or unloading $(d \gamma<0)$ :

$$
\begin{array}{rlrl}
G_{\gamma}^{+}=\frac{\partial \tau}{\partial \gamma}_{u, d \gamma>0} & G_{\gamma}^{-}=\frac{\partial \tau}{\partial \gamma} \\
N_{\gamma}^{+}=\frac{\partial \sigma}{\partial \gamma}_{u, d \gamma>0} & N_{\gamma}^{-}=\frac{\partial \sigma}{\partial \gamma} \\
u, d \gamma<0
\end{array}
$$


Second, a constant tangential displacement (CTD) path, with $(d u=c s t, d \gamma=0)$ is used. Four other moduli are defined from the resulting curves $\tau(u)$ and $\sigma(u)$, considering both loading $(d u>0)$ or unloading $(d u<0)$ :

$$
\begin{aligned}
G_{u}^{+}=\frac{\partial \tau}{\partial u}_{\gamma, d u>0} & G_{u}^{-}=\frac{\partial \tau}{\partial u}_{\gamma, d u<0} \\
N_{u}^{+}=\frac{\partial \sigma}{\partial u}_{\gamma, d u>0} & N_{u}^{-}=\frac{\partial \sigma}{\partial u}{ }_{\gamma, d u<0}
\end{aligned}
$$

These moduli are identical for both relations and determine completely the behaviour along the two calibration paths. Both relations are thus identical along such paths. The behaviours along any other loading paths are defined from the behaviours along calibration paths through two different interpolations. Finally a quadrilinear formalism expresses the response of the joint according to Quadri relation :

$$
\left(\begin{array}{c}
d \tau \\
d \sigma
\end{array}\right)=\frac{1}{2}\left(P^{+}+P^{-}\right)\left(\begin{array}{c}
d \gamma \\
d u
\end{array}\right)+\frac{1}{2}\left(P^{+}-P^{-}\right)\left(\begin{array}{l}
|d \gamma| \\
|d u|
\end{array}\right)
$$

With matrices $P^{+}$and $P^{-}$depending on the eight previous moduli such as :

$$
P^{+}=\left(\begin{array}{cc}
G_{\gamma}^{+} & G_{u}^{+} \\
N_{\gamma}^{+} & N_{u}^{+}
\end{array}\right) \quad P^{-}=\left(\begin{array}{cc}
G_{\gamma}^{-} & G_{u}^{-} \\
N_{\gamma}^{-} & N_{u}^{-}
\end{array}\right)
$$

Whereas, for INL2 relation, the formalism is incrementally non linear of second order :

$$
\left(\begin{array}{c}
d \tau \\
d \sigma
\end{array}\right)=\frac{1}{2}\left(P^{+}+P^{-}\right)\left(\begin{array}{c}
d \gamma \\
d u
\end{array}\right)+\frac{1}{2 \sqrt{d u^{2}+d \gamma^{2}}}\left(P^{+}-P^{-}\right)\left(\begin{array}{c}
d \gamma^{2} \\
d u^{2}
\end{array}\right)
$$

Detailed expressions for the moduli were proposed in [13]. They involve some parameters which are listed in Table 2, with their values calibrated in [13] for infilled rock joints. In this Table, the parameters are grouped according to their

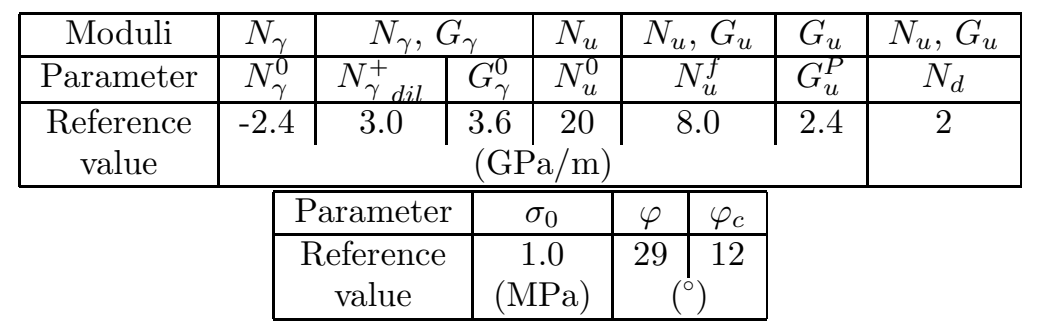

TABLE 2 - Default values of Quadri or INL2 relations parameters [13]

corresponding moduli (moduli " $+"$ and " $-"$ are here not distinguished). Some parameters correspond to different moduli because some links between moduli were introduced here in order to obey Equation (2). Following section 2.3 gives some details. 
These two relations correspond to the INL and octolinear constitutive relations developped by Darve for soils [42, 43]. Quadri and INL2 relations were compared in [13] and, as it is the case for the soils relations, they predict close behaviours and present same general properties. Thanks to expressions of moduli proposed in [13] both respect a plastic limit criterion : the stress states they predict can not exceed a Coulomb like criterion : $\tau=\sigma \tan (\varphi)$. Both are also nonassociated (their flow rules were determined in [13]). This motivates the use of the second order work criterion.

\subsection{Bifurcation domains and directions of instability for Quadri and INL2 relations}

\subsubsection{Bifurcation domains}

For Quadri case, equation of bifurcation domain, Equation (10), can be directly computed, for each matrix of each tensorial zone. After this step, and after having verified that directions of instability belong to the right tensorial zone, a bifurcation domain for $(d \gamma>0, d u<0)$ tensorial zone is found. Its limits are plotted in Mohr-plane in Figure 2.

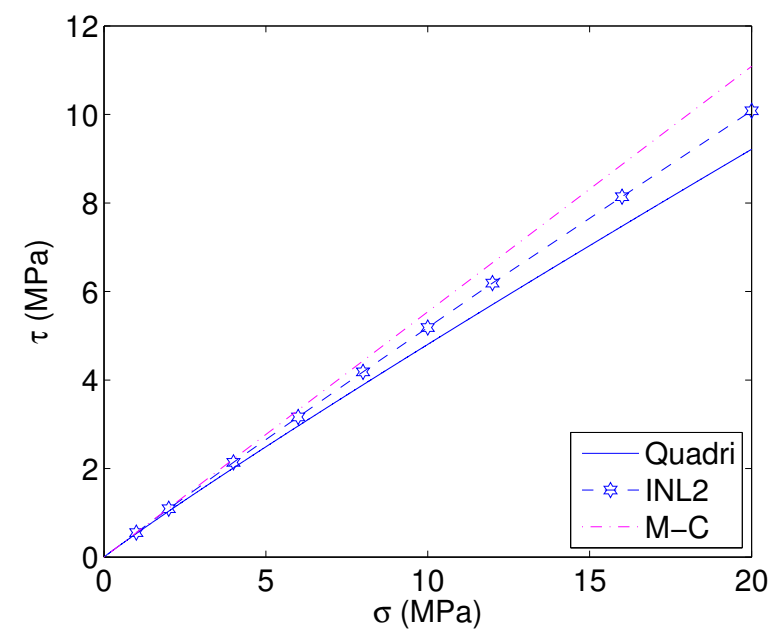

Figure 2 - Bifurcation domains of Quadri and INL2 constitutive relations

Figure 2 also represents the limit for INL2 constitutive relation. In this case, no analytical development can be done. To detect the mechanical states belonging to the bifurcation domain of INL2 relation, a "directionnal analysis" is performed, as in [52] or [24] for example. For a sufficient number of mechanical states (discretizing the Mohr plane), different incremental loadings $\overrightarrow{d l}$ are considered, with different directions $\theta d l$ (see Figure 3). Corresponding response $\overrightarrow{d \sigma}$ 


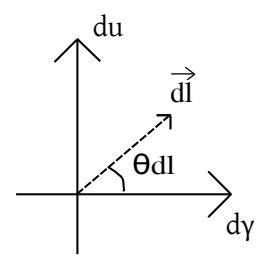

Figure 3 - Definition of incremental displacement direction, $\theta d l$

and resulting second order work $d^{2} w$ are computed. If $d^{2} w \leqslant 0$ for some directions $\theta d l$, the mechanical state belongs to the bifurcation domain. This domain is thus identified, and its limits can be plotted, see Figure 2.

Both bifurcation domains concern sheared states of the joint, more or less close to Mohr-Coulomb criterion. Shearing rock joint appears to be as a catalyst of instability. From Equation (10), this can be understood because during the shearing deformation diagonal rigidities decrease [1] whereas nondiagonal ones, as moduli $G_{u}[10,13]$, increase. Differences between bifurcation domains and plastic limit criterion increase with $\sigma$. Influence of $\sigma$ on the mechanical behaviour of rock joint is also well known regarding the normal stiffness of the joint $[1,2,4]$. When comparing INL2 with Quadri cases, the bifurcation domain is greater in case of the Quadri constitutive relation. This was already observed for soils framework, using octolinear and INL relations [48].

\subsubsection{Instability cones}

Figure 4 presents directions of instability for the Quadri case, for different $\tau / \sigma=\tan \left(\phi_{m o b}\right)$ ratios, which correspond to different states in shearing of the joint. In the displacement plane, directions of instability imply shear loading

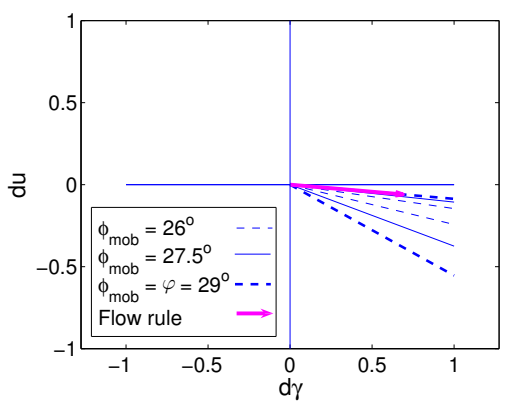

(a) Displacement plane

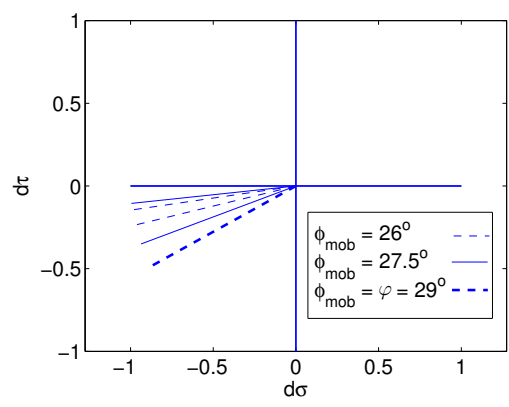

(b) Mohr plane

FIgURE 4 - Unstable directions in Quadri case : influence of shearing. Under $\sigma=10 \mathrm{MPa}$, for different $\tau$ values $\left(\tau=\sigma \tan \left(\phi_{m o b}\right)\right)$

$(d \gamma>0)$, associated with a slight dilatancy $(d u<0)$. This result was also 
obtained for a contact between two particles of a granular material, by Nicot and Darve [25], from a micro-mechanical point of view. Regarding stresses, directions of instability correspond to decreases for both stresses : $d \tau$ and $d \sigma$ are negative. According to the discussion developed in paragraph 1.2 this is because $G_{u}^{-}<N_{\gamma}^{+}$ at these mechanical states.

For different shearing states, inside the bifurcation domain and before reaching plastic limit criterion, cones are more open if $\phi_{m o b}$ increases, either in displacement or stress planes. Such results were already obtained for soils relations in 2D (axisymmetric) [14] or 3D conditions [48], and using a micro-mechanical approach or direct numerical simulations [47] with a discrete element method. Once the stress state is on the plastic limit criterion, two remarks can be made.

First, one of the branchs of the displacement cone corresponds to the flow rule of the material, which is also plotted in Figure 4(a). Using our relations, which do not distinguish elastic and plastic parts of strains (relative displacement here), the flow rule is defined as the direction of the relative displacements leading to $\overrightarrow{d \sigma}=\overrightarrow{0}$, on the plastic limit criterion [13] (or see [53] for comparable soil relations). It corresponds to the eigenvector of eigenvalue 0 , of the constitutive matrix on plastic limit criterion since its determinant vanishes for this criterion. Such couple $(\overrightarrow{d l} \neq \overrightarrow{0}$ given by flow rule, $\overrightarrow{d \sigma}=\overrightarrow{0})$ leads to $d^{2} w=\overrightarrow{d l} \cdot \overrightarrow{d \sigma}=0$.

Second remark concerns unstable stress directions. Figure 4(b) shows that, on plastic criterion, the unstable stress direction cone reduces finally in an halfline, parallel to Mohr-Coulomb criterion $\left(d \tau / d \sigma=\tan (\varphi)=\tan \left(29^{\circ}\right)\right.$ here $)$. The unstable stress direction linked with flow rule is indeed not defined $(\overrightarrow{d \sigma}=\overrightarrow{0})$, and all other unstable displacement directions correspond to this descending branch of Mohr-Coulomb criterion, as it was shown in [13]. Note that other stress directions which would point outward the Mohr-Coulomb criterion are constitutively not admissible, since that through the constitutive relation they do not correspond to any displacement direction [13]. Consequently, such stress directions are not considered here.

Influence of $\sigma$, for a given shearing state, on Quadri-case directions of instability can be observed in Figure 5 . As it was the case for increasing shearing, cones get also more open for increasing $\sigma$ values.

Unstable directions for INL2 relation are presented, and compared to those of Quadri relation in Figure 6. Both types of cones are comparable, belonging to the same quadrants of displacement and Mohr planes : $(d \gamma>0, d u<0)$

and $(d \tau<0, d \sigma<0)$ (Figure 6 zooms on these quadrants). INL2 cones extend nevertheless less than Quadri ones.

\subsubsection{Loss of definite positiveness of the symmetric part of consti- tutive matrix}

In the previous section 1.3 it was explained why the set of directions of instability is included inside the set of directions which let $M^{S}$ having a negative determinant, for constitutive relations with several tensorial zones. This result 


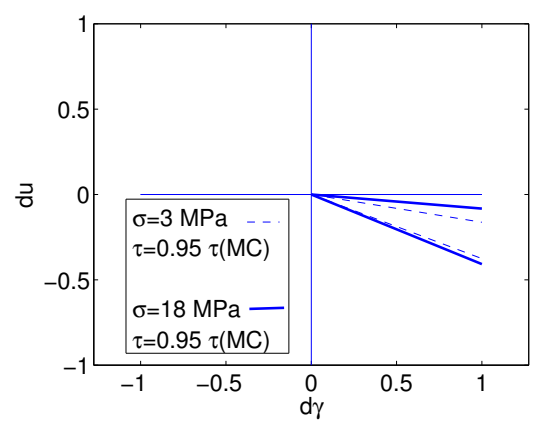

(a) Displacement plane

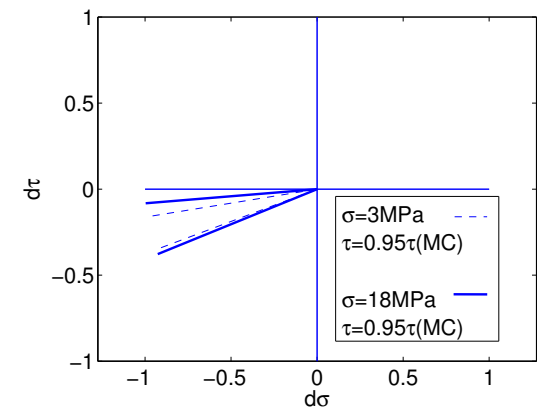

(b) Mohr plane

Figure 5 - Unstable directions in Quadri case : influence of $\sigma$. For $\tau$ values close to $\tau(\mathrm{MC})=\sigma \tan (\varphi)$, the maximal value corresponding to Mohr-Coulomb plastic limit criterion

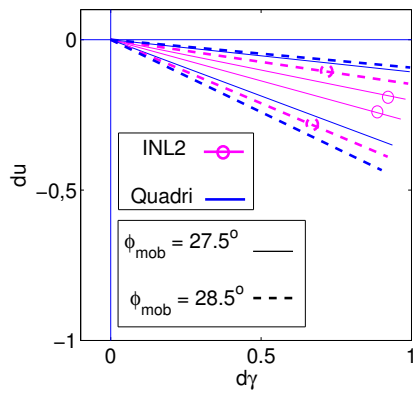

(a) Displacement plane

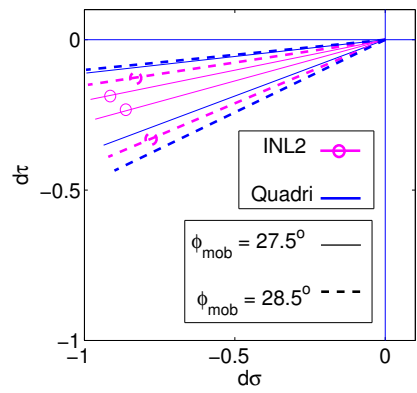

(b) Mohr plane

FIgURE 6 - Comparison of directions of instability between Quadri and INL2 relations. For $\sigma=10 \mathrm{MPa}$ 
is now illustrated for our Quadri and INL2 relations. Note that $\operatorname{det}\left(M^{S}\right)$ varies among four values for Quadri case, or in a continuous manner for INL2 case.

For a given mechanical state belonging to the bifurcation domain, the symmetric parts of the two constitutive matrices corresponding to Quadri and INL2 relations are computed according to the loading direction, and their determinants are plotted in Figure 7 according to $\theta d l$ (which expresses the loading direction, see previous Figure 3). On Figure 7(a) cartesian representations are adopted. The piecewise constant, discontinuous, values for $\operatorname{det}\left(M^{S}\right)$ appear in Quadri case, whereas the variations are continuous for INL2 case. Nevertheless, the values are comparable between the two cases. For Quadri case, $\operatorname{det}\left(M^{S}\right)$ is negative only for $\theta d l \in\left[270^{\circ} ; 360^{\circ}\right]$, i.e. in $(d \gamma>0, d u<0)$ tensorial zone, where the directions of instability were indeed found : for $\theta d l \in\left[338^{\circ} ; 352^{\circ}\right]$.

For INL2 case, considering Figure $7(\mathrm{~b})$ is more convenient. On this Figure a circular representation is adopted, i.e. a polar representation of $d M s=$ $A+\operatorname{det}\left(M^{S}\right)$, with $A$ chosen such that $d M s$ remains stricly positive, even if $\operatorname{det}\left(M^{S}\right) \in[-20 ; 50](\mathrm{MPa} / \mathrm{mm})^{2}$. Negative values of $\operatorname{det}\left(M^{S}\right)$ can be observed on this Figure, where the corresponding curve passes inside the reference circle $d M s=A \Leftrightarrow \operatorname{det}\left(M^{S}\right)=0$. Here again, the directions of instability belong to the set of directions leading to $\operatorname{det}\left(M^{S}\right) \leqslant 0$, as it can be observed if the instability cone is plotted on the same Figure $7(\mathrm{~b})$.

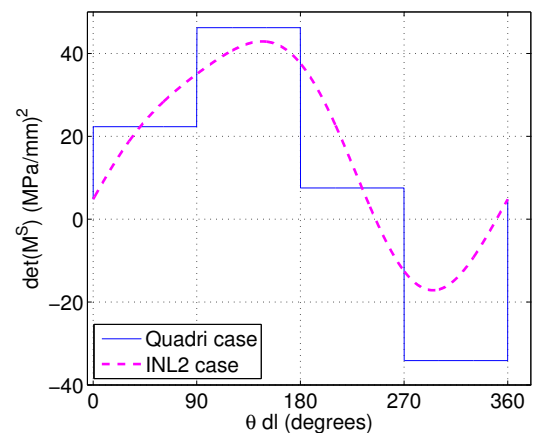

(a) Cartesian representation of $\operatorname{det}\left(M^{S}(\theta d l)\right)$ (b) Circular representation of $\operatorname{det}\left(M^{S}(\theta d l)\right)$ for Quadri and INL2 cases

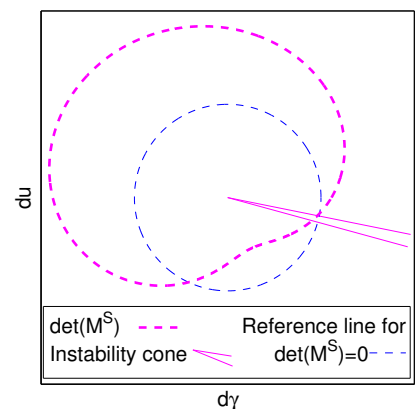

for INL2 case, compared with instability cone

FiguRe 7 - Directional variations of the determinant of the symmetric parts of constitutive matrices (Quadri and INL2 cases). For $\sigma=10 \mathrm{MPa}$, and $\tau=$ $\sigma \tan \left(27.5^{\circ}\right)$ 


\subsection{Quadri case : sensitivity analysis of the bifurcation domain}

Expressions of moduli of Quadri and INL2 relations, hence their bifurcation domains, depend on the parameters which were listed in Table 2. Following paragraphs illustrate the sensitivity of the bifurcation domain depending on some of these parameters.

Doing so, we also show how bifurcation domains can be modified for different rock joints with different normal rigidities (different moduli $N_{u}$ ), tangential rigidities $\left(G_{\gamma}\right)$, or dilatant feature $\left(N_{\gamma}\right)$, etc... Since bifurcation domains of Quadri and INL2 relations are close, only the Quadri one will be considered. Recall that Equation (10) is the equation of this domain.

\subsubsection{Moduli $N_{u}$ : parameters $N_{u}^{0}$ and $N_{u}^{f}$}

Moduli $N_{u}^{+/-}$correspond to normal stiffnesses, in loading or unloading, of the joint along CTD $(d \gamma=0)$ paths. The formulation (expressed in details in [13]) involves two parameters : $N_{u}^{0}$ and $N_{u}^{f}$ which rule the values of the normal stifnesses. These stiffnesses increase with $\sigma$, proportionaly to $N_{u}^{0}$ for nonsheared states $(\tau=0)$, or to $N_{u}^{f}$ for completely sheared states $(\tau=\sigma \tan (\varphi))$, or to intermediate values between $N_{u}^{0}$ and $N_{u}^{f}$ for intermediate shearing states. Thus, moduli $N_{u}^{+/-}$are increasing while $N_{u}^{0}$ and/or $N_{u}^{f}$ increase. Considering Equation (10), increase in normal rigidity tends to reduce the size of the bifurcation domain. However, parameter $N_{u}^{f}$ is also used as a multiplying parameter in expression of moduli $G_{u}^{-}$and higher moduli $G_{u}^{-}$should increase the size of the bifurcation domain. Because of these two contradictory effects, the influence of parameter $N_{u}^{f}$ is not easily predictable using Equation (10). This approach is now detailled, considering both parameters apart first, before considering changes in the two parameters at the same time.

- $N_{u}^{0} \quad$ Decrease in $N_{u}^{0}$ value corresponds to rock joints with lower normal stiffnesses (along CTD paths), under non- (or few-) sheared states. Thus, decreasing values of $N_{u}^{0}$ increase the bifurcation domain, see Figure 8.

- $N_{u}^{f} \quad$ For higher values of $N_{u}^{f}$ (limited by $N_{u}^{0}=20 \mathrm{GPa} / \mathrm{m}$ ), the normal stiffnesses of the joint $N_{u}$ less decreases during shearing, which tends to reduce the bifurcation domain. But, a link exists between $N_{u}^{f}$ and the final value of $G_{u}^{-}$(on the Mohr-Coulomb criterion) and higher values of $N_{u}^{f}$ also increase $G_{u}^{-}$ moduli. Hence, the consequences on the bifurcation domain limit are difficult to predict using only Equation (10).

However, considering the ratio $G_{u} / N_{\gamma}$ is useful. This ratio is equal to one for symmetric matrices which correspond to associated behaviours, hence, the bifurcation domain strictly equal to the plastic limit. For the reference case $\left(N_{u}^{f}=8 \mathrm{GPa} / \mathrm{m}\right)$, under $\sigma=10 \mathrm{MPa}$ and $\tau=\sigma \tan \left(20^{\circ}\right)$, we have $G_{u}^{-} / N_{\gamma}^{+} \approx 40$. This ratio is actually proportionnal to $N_{u}^{f}$, thus higher values of $N_{u}^{f}$ increase it, 


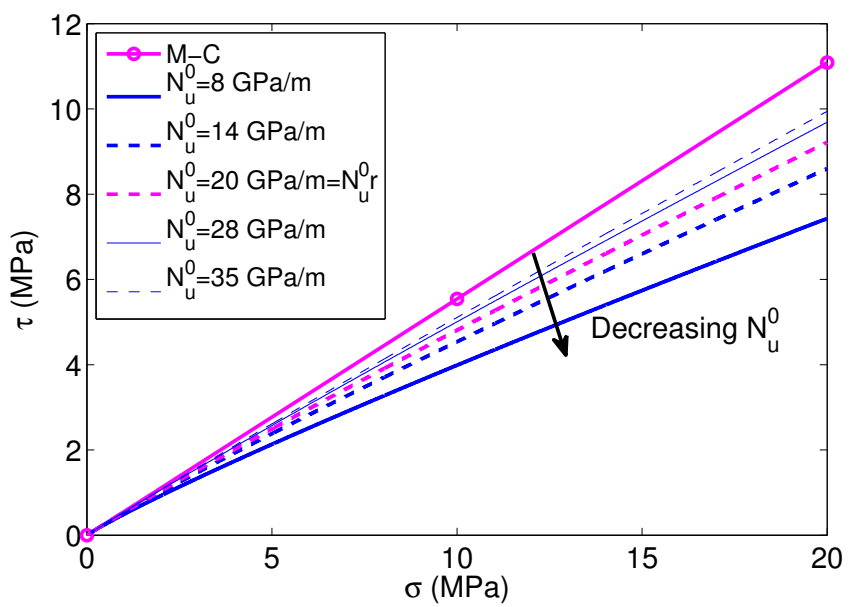

FiguRE 8 - Quadri bifurcation domain : Influence of $N_{u}^{0}$ on its limit $\left(N_{u}^{0} r=\right.$ $20 \mathrm{GPa} / \mathrm{m})$

away from the value of 1 , e.g. around 100 for $N_{u}^{f}=20 \mathrm{GPa} / \mathrm{m}$ under the same stress state. The nonassociativity of the behaviour is more marked, leading to a wider bifurcation domain. This is confirmed by Figure 9 : for the tested values, the bifurcation domain grows with $N_{u}^{f}$.

$-N_{u}^{0}, N_{u}^{f} \quad$ Finally, changes in both $N_{u}^{0}$ and $N_{u}^{f}$ are considered. We just derived that increases of these two parameters have opposite influences on the bifurcation domain. The Figure 10 presents the case for tested values of $N=\left(N_{u}^{0} ; N_{u}^{f}\right)$, compared to the reference value $N r=\left(N_{u}^{0}=20 \mathrm{GPa} / \mathrm{m} ; N_{u}^{f}=8 \mathrm{GPa} / \mathrm{m}\right)$. The influence of parameter $N_{u}^{f}$ appears to be preponderant : for the range of tested values, we observe that the bifurcation domain increases depending on $N$ values.

\subsubsection{Moduli $G_{\gamma}$ : parameter $G_{\gamma}^{0}$}

Tangential rigidity $G_{\gamma}\left(G_{\gamma}^{+}\right.$for loading, $G_{\gamma}^{-}$for unloading $)$is now considered. Values of $G_{\gamma}^{+}$decrease during shearing from an initial value $G_{\gamma}^{0}$ (for $\tau=0$ ) to a final value $G_{\gamma}^{f}$ (for $\tau=\sigma \tan (\varphi)$ ). Considering an elastic unloading, the initial value $G_{\gamma}^{0}$ also serves as constant value for $G_{\gamma}^{-} . G_{\gamma}^{f}$ value depends on parameter $N_{\gamma \text { dil }}^{+}$which will be considered in next section. Thereby, only changes in $G_{\gamma}^{0}$ are considered, see Figure 11. All values are chosen to be greater than the final value $G_{\gamma}^{f}=N_{\gamma}^{+}{ }_{d i l} \tan (\varphi) \approx 1.66 \mathrm{GPa} / \mathrm{m}$. Decreasing values of $G_{\gamma}^{0}$ increase the size of the bifurcation domain, as expected from Equation (10); but the influence is much less important than for other parameters. 


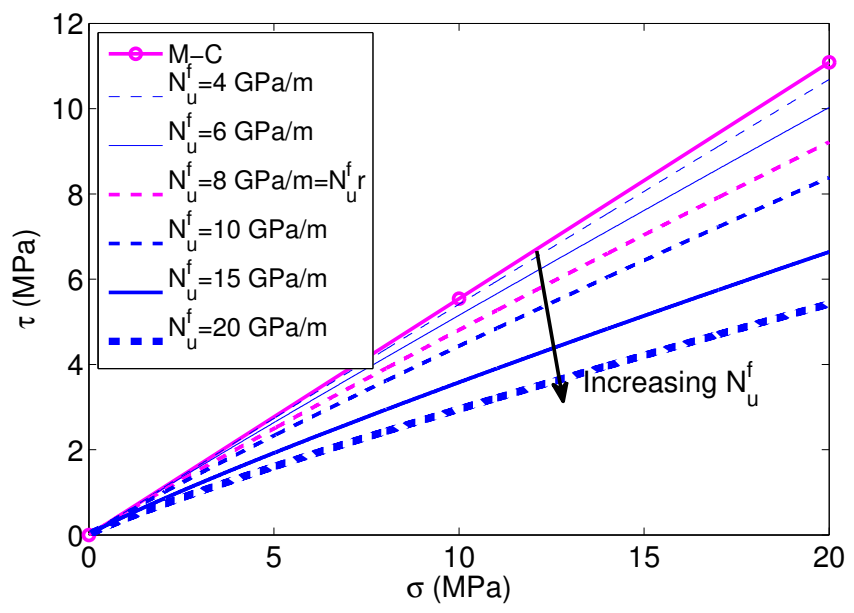

Figure 9 - Quadri bifurcation domain : Influence of $N_{u}^{f}$ on its limit (reference $\left.N_{u}^{f} r=8 G P a / m\right)$

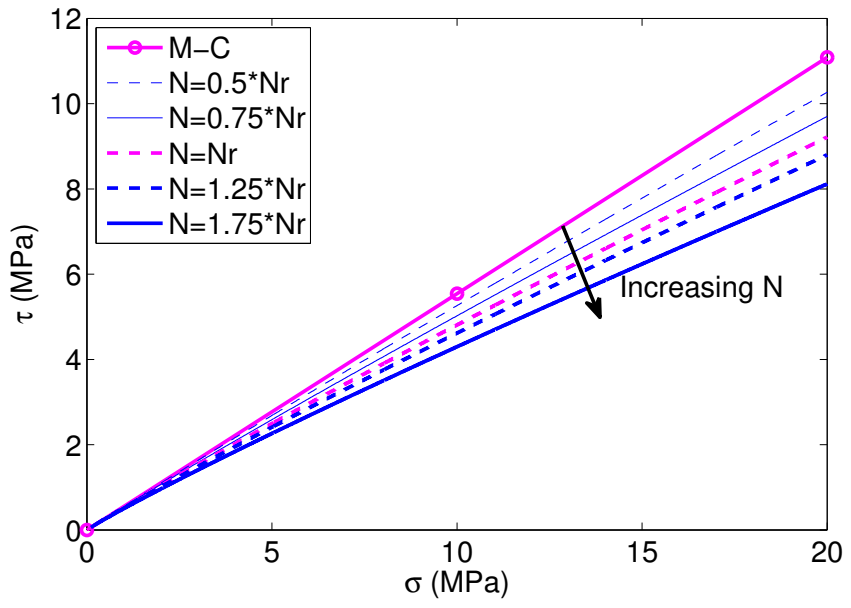

FIgURE 10 - Quadri bifurcation domain : Influence of $N=\left(N_{u}^{0} ; N_{u}^{f}\right)$ on its $\operatorname{limit}(N r=(20 \mathrm{GPa} / \mathrm{m} ; 8 \mathrm{GPa} / \mathrm{m}))$ 


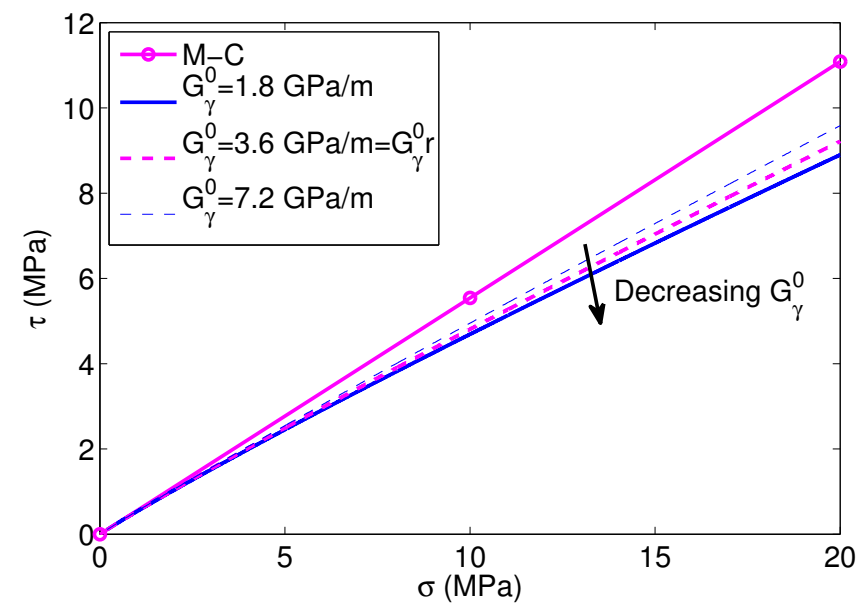

FIgURE 11 - Quadri bifurcation domain : Influence of $G_{\gamma}^{0}$ on its limit (reference $\left.G_{\gamma}^{0} r=3.6 \mathrm{GPa} / \mathrm{m}\right)$

\subsubsection{Moduli $N_{\gamma}$ : parameter $N_{\gamma}^{+}$dil}

In order to reproduce a behaviour which is first contractant before being dilatant, two parameters are used : $N_{\gamma}^{0}<0$ and $N_{\gamma}^{+}{ }_{d i l}>0$. Modulus $N_{\gamma}^{+}$is first equal to parameter $N_{\gamma}^{0}$ (whose influence is not considered here), and second reaches $N_{\gamma}^{+}$dil value. Figure 12 considers different values for parameter $N_{\gamma}^{+}{ }_{d i l}$, which first corresponds to the dilatant feature of the joint. Hence, increasing $N_{\gamma}^{+}$dil should let increase the bifurcation domain. This parameter also controls the values of tangential rigidity for sheared state, and, increasing tangential rigidity decreases the bifurcation domain. Figure 12 shows that the influence of tangential rigidity is preponderant : the bifurcation domain increases while $N_{\gamma \text { dil }}^{+}$decreases.

Again, considering the ratio $G_{u}^{-} / N_{\gamma}^{+}$explains the influence. This ratio is actually proportionnal to $1 / N_{\gamma}^{+}$dil . For example, for the reference case with $\sigma=10 \mathrm{MPa}$ and $\tau=\sigma \tan \left(20^{\circ}\right), G_{u}^{-} / N_{\gamma}^{+} \approx 40$. Whereas $G_{u}^{-} / N_{\gamma}^{+} \approx 80$ for $N_{\gamma}^{+}{ }_{d i l}=1.5 \mathrm{GPa} / \mathrm{m}$. Hence, decreasing $N_{\gamma}^{+}$dil moves away the value of the ratio from one, leading to a wider bifurcation domain.

\subsection{A convenient set of parameters}

In the following sections 3 and 4 the rock joint behaviour is studied when the second order work criterion is fulfilled. To perform this task, a new set of parameters is considered, different from the one of Table 2 which corresponds to Quadri and INL2 relations presented in [13]. These new values of parameters are presented in Table 3. Parameter $N_{u}^{f}$ is the only one to be modified, and is 


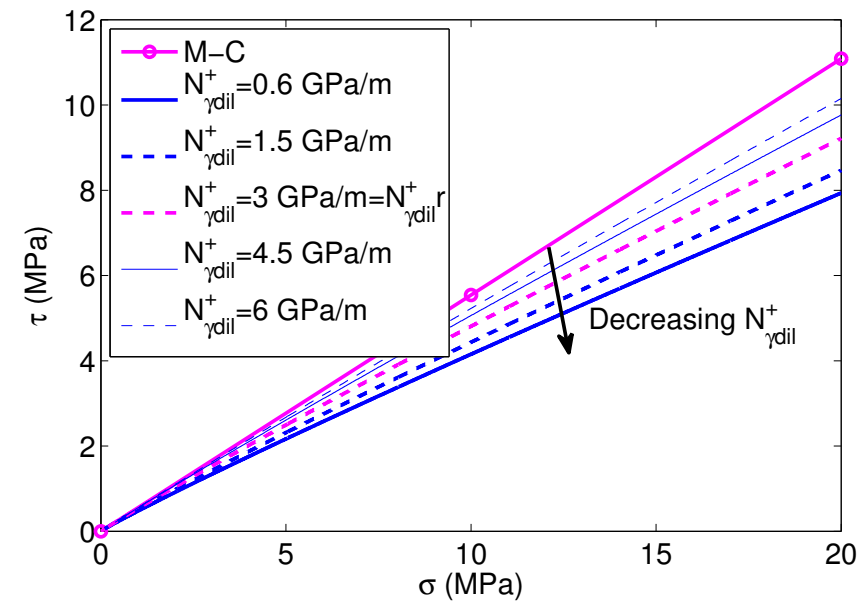

FIGURE 12 - Quadri bifurcation domain : Influence of $N_{\gamma}^{+}$dil on its limit (reference $\left.N_{\gamma}^{+}{ }_{d i l} r=3.0 \mathrm{GPa} / \mathrm{m}\right)$

\begin{tabular}{|c|c|c|c|c|c|c|c|c|c|}
\hline \multicolumn{6}{|c|}{ (GPa } & \multicolumn{2}{|c|}{$(\mathrm{MPa})$} & \\
\hline$N_{\gamma}^{0}$ & $N_{\gamma}^{+}$ & $G_{\gamma}^{0}$ & $N_{u}^{0}$ & $\mathbf{N}_{\mathbf{u}}^{\mathrm{f}}$ & $G_{u}^{P}$ & $N_{d}$ & $\sigma_{0}$ & $\varphi$ & $\varphi_{c}$ \\
\hline$\frac{1}{-2.4}$ & 3.0 & 3.6 & 20 & 15.0 & 2.4 & 2 & 1.0 & 29 & 12 \\
\hline
\end{tabular}

TABLE 3 - Set of parameters "SetU", corresponding to a wider bifurcation domain 
considered equal to $15.0 \mathrm{GPa} / \mathrm{m}$, instead of $8.0 \mathrm{GPa} / \mathrm{m}$ previously. This corresponds to two features of the joint : the corresponding rock joint presents a less important decrease in normal rigidity with shearing stresses; and moduli $G_{u}^{-}$can reach greater values, during shearing. A more important bifurcation domain is obtained. The frontier of the domain corresponds to $\tau / \sigma \approx \tan \left(18.4^{\circ}\right)$ for $\sigma=15 \mathrm{MPa}$ (see Figure 9), instead of $\tau / \sigma \approx \tan \left(25.2^{\circ}\right.$ ) previously (see Figure 2 ). Cones of directions of instability are also wider (Figure 13 compared with $4)$.

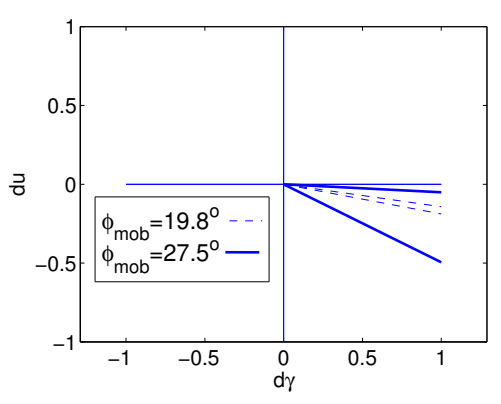

(a) Displacement plane

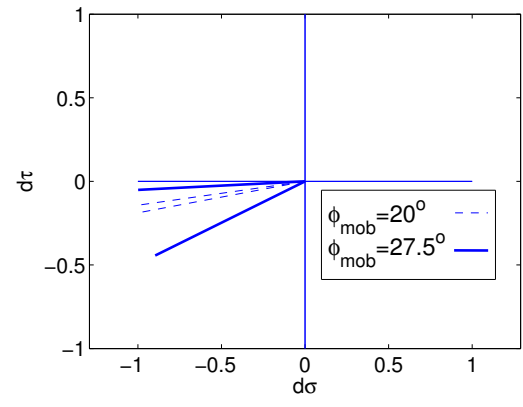

(b) Mohr plane

Figure 13 - Unstable directions for set of parameters "SetU", under $\sigma=10 \mathrm{MPa}$

A plastic limit criterion (Mohr-Coulomb, with $c=0 \mathrm{~Pa}$, and $\varphi=29^{\circ}$ ) is still obtained : this feature does not depend on the parameters values of our relations [13]. To justify the use of the second order work criterion with these new parameter values, it has to be checked that the behaviour is still nonassociated. In a previous work [13], a dilatancy angle $\psi$ was defined to control the flow rule, which expresses the direction of relative displacements $\overrightarrow{d l}^{p}=\left(d u^{p}, d \gamma^{p}\right)$ occuring once stresses do not evolve on plastic limit criterion, with $\tan (\psi)=d u^{p} / d \gamma^{p}$. Values of $\psi$ for Quadri and INL2 relations were computed for the reference parameters presented in Table 2 in [13] : they were close and different from $\varphi$ for both relations. For the new set of parameter of Table $3, \psi$ values (for Quadri relation) are presented in Figure 14 : they are still different from $\varphi=29^{\circ}$ (and decreasing according to $\sigma$ ) : the behaviour is still nonassociated.

\section{Displacement proportional loading paths}

Sections 3 and 4 illustrate conditions for which $d^{2} w \leqslant 0$, and compare the rock joint behaviour to soil behaviour, for which experimental evidences of negative second order work (and corresponding failures) were observed. In order to study conditions for which $d^{2} w \leqslant 0$, it is convenient to consider proportional loading paths. In soil case, in axisymmetric conditions, we define strain proportional loading paths as given by : $d \varepsilon_{1}+2 R d \varepsilon_{3}=0$, with $R$ fixed for one test 


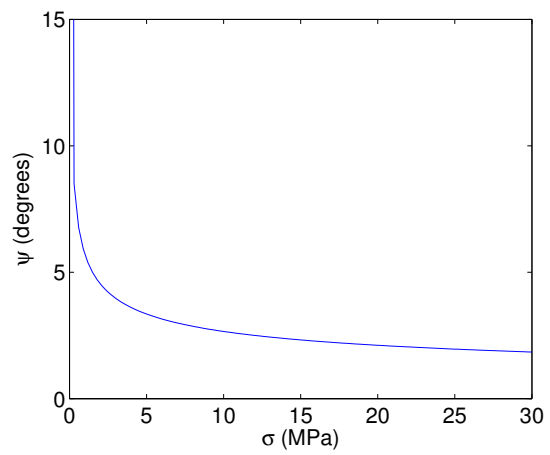

Figure 14 - Dilatancy angle values for parameters SetU

path. The undrained (i.e. isochoric) triaxial test with $R=1$ is an example.

For rock joints, displacement proportional loading paths are controled by $d u+k d \gamma=0$, with $k$ constant. For $k$ positive, a dilatant behaviour is imposed, for $k$ negative it is a contractant one. Along such paths note that:

$$
d^{2} w=d \sigma d u+d \tau d \gamma=(d u+k d \gamma) d \sigma+(d \tau-k d \sigma) d \gamma=(d \tau-k d \sigma) d \gamma
$$

Equation (21) shows that the second control variable for such paths, in addition to $d u+k d \gamma=0$, can be either $d(\tau-k \sigma)=c s t$, or $d \gamma=c s t$, since these two variables appear to be conjuguated with respect to energy. Complete control parameters of such paths are resumed in Equation (22).

$$
\left\{\begin{array}{c}
d u+k d \gamma=0 \\
d \gamma=c s t \text { or } d \tau-k d \sigma=c s t
\end{array}\right.
$$

Moreover Equation (21) shows that, along such paths :

$$
d^{2} w=0 \Leftrightarrow\left\{\begin{array}{l}
d(\tau-k \sigma)=0 \text { if } d \gamma \text { is imposed } \\
\text { or } d \gamma=0 \text { if } d(\tau-k \sigma) \text { is imposed }
\end{array}\right.
$$

For the case where $d \gamma$ is imposed, instability is obtained when $\tau-k \sigma$ passes through a maximum and decreases. In three dimensions, Darve et al. [14] derived a similar condition :

$$
d^{2} w=0 \Leftrightarrow d\left(\sigma_{1}-\sigma_{3} / R\right)=0
$$

These results are now applied to various paths, with given values of $k$, considering first the constant volume shear case $(k=0)$.

\subsection{Constant volume shear : CND path}

For soils, constant volume shear occurs during undrained triaxial experiments. For experiments involving loose sand a peak of $q$ is obtained before Mohr-Coulomb criterion. This peak is unstable and could lead to failures that 
can be explained only using the second order work $[14,15]$. For rock joints a constant volume shear implies $d u=0$, which corresponds to CND paths and to a vanishing value of control parameter $k$ in Equation (22). The instability cones previously presented in Figures 4(a), for some values of parameters, did not show this loading direction $(d u=0, d \gamma)$ as an unstable one. Let us now consider any Quadri or INL2 relations along such paths, theoretically and then numerically. For these loading conditions there is no difference between the two relations.

\subsubsection{Theoretical discussion}

For both relations, with $d u=0$ :

$$
d^{2} w=d \sigma d u+d \tau d \gamma=d \tau d \gamma
$$

Either $d \tau$ or $d \gamma$ is the second loading parameter (see previous Equation (22)). Both are directly linked by moduli $G_{\gamma}$, by definition along this calibration path. For such path, instability occurs when the following condition is met :

$$
d^{2} w \leqslant 0 \Leftrightarrow G_{\gamma}^{+/-} \leqslant 0
$$

According to the calibration data [10], strictly positive values for $G_{\gamma}^{+/-}$are used. In this case, failure of rock joints before plastic limit condition during constant volume (or undrained) conditions does not occur. It would for negative values of $G_{\gamma}^{+/-}$: decreases in $\tau$ according to $\gamma$ along CND paths. This corresponds directly to soils' case, where instability is also obtained when the deviatoric part of the stress $(\propto q)$ decreases depending on the deviatoric strains $\left(\propto \varepsilon_{1}\right.$ for undrained tests).

\subsubsection{Numerical results}

In order to illustrate the previous analytical developments, CND paths are performed with INL2 or Quadri relations. Increments $(d u=0, d \gamma=$ cst) are imposed, and stress responses can be determined either with Equation (20) or (18) (recall that the results are here identical). For each loading increment, the second order work is computed, and its evolution during the loading is considered. The value of $d \gamma$ must be small enough to have no influence on the results (see Figures 15(a) and 15(b)). Even if differences on stress values vanish, magnitude of computed second order work values still depend highly on $d \gamma$ value : see Figure $15(\mathrm{c})$ for $d \gamma=0.05 \mathrm{~mm}$ and $0.001 \mathrm{~mm}$ cases, for which stress path are equal. This can be avoided with the use of a normalised second order work $d^{2} w^{N}$, such as :

$$
d^{2} w^{N}=\frac{d^{2} w}{\|\overrightarrow{d \sigma}\|\|\overrightarrow{d l}\|}=\frac{d \sigma d u+d \tau d \gamma}{\sqrt{d \sigma^{2}+d \tau^{2}} \sqrt{d u^{2}+d \gamma^{2}}}
$$

Signs of $d^{2} w^{N}$ and $d^{2} w$ are the same, and Figure 15(d) shows that comparable 


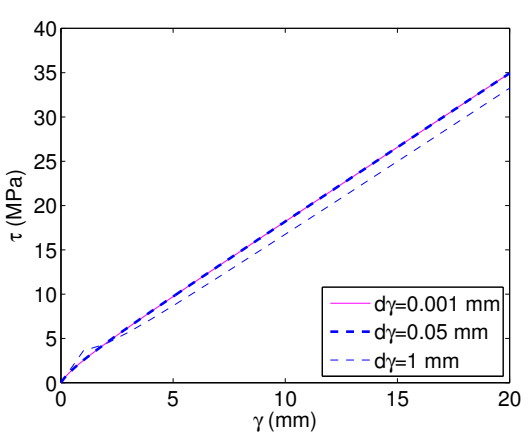

(a) $\tau(\gamma)$

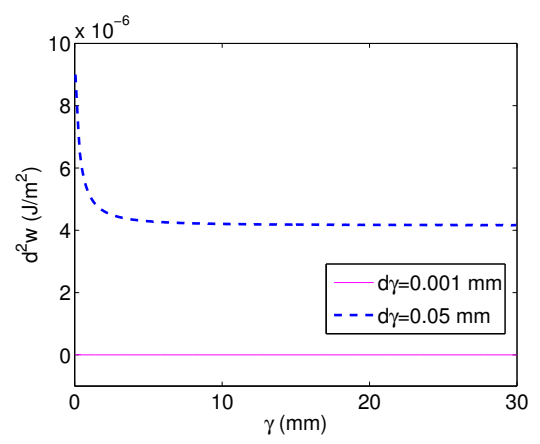

(c) $d^{2} w$

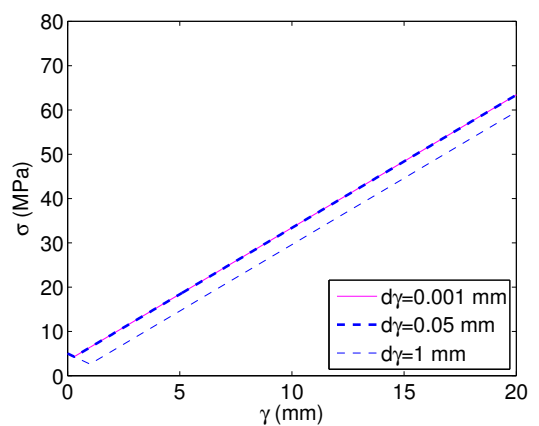

(b) $\sigma(\gamma)$

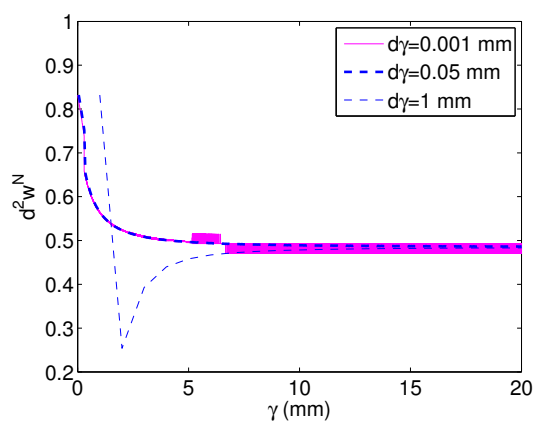

(d) Normalised second order work $d^{2} w^{N}$

Figure 15 - Influence of $d \gamma$ value for INL2 (= Quadri here) CND tests 
values are obtained, which do not evolve anymore once $d \gamma$ is small enough. However, because of numerical precision issues, too small values of $d \gamma$ could introduce noise in the data : cases $d \gamma=0.05 \mathrm{~mm}$ and $0.001 \mathrm{~mm}$ present same $d^{2} w^{N}$ values in average, but with some noise for $d \gamma=0.001 \mathrm{~mm}$.

Let us now follow the evolution of $d^{2} w^{N}$ during loading. Initially positive, a decrease in normalized second order work is observed (Figure 15(d)), but not enough to reach nil or negative values in this case. A plateau for $d^{2} w^{N}$ is reached.

Comparison between these results with a second CND path (from a different initial normal stress $\sigma_{0}$ ) is presented in Figure 16. In Figure 16(b), the normalized second order work is plotted according to $\tau / \sigma$ (whose values are limited by $\tan (\varphi)$ ), rather than $\gamma$. Decreases are still observed. Sharp decreases occur for $\tau / \sigma=\tan \left(\varphi_{c}\right) \approx 0.21$ when the $N_{\gamma}^{+}$moduli changes in a discontinuous manner. Plateau of $d^{2} w^{N}$ does not appear anymore in Figure 16(b), which means that the plateau in Figure 15(d) corresponds to the one of $\tau / \sigma$ which is reached along such paths : an asympotic point, rather than an asymptotic line, exists in Figure 16(b).

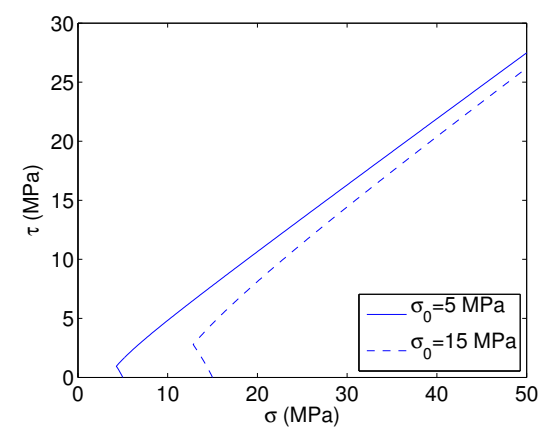

(a) $\tau(\sigma)$

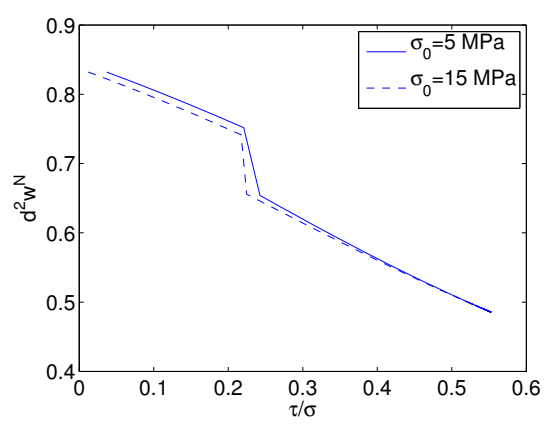

(b) $d^{2} w^{N}(\tau / \sigma)$

FiguRE 16 - Two different DNC paths performed with Quadri relation (=INL2 here)

\subsection{Other displacement proportional loading paths}

Other $k$ values are now considered, which especially correspond to directions of instability. From Figure 13, we can deduce that, for example, $d u / d \gamma \approx$ $-\tan \left(15^{\circ}\right)$ is a direction of instability for $\sigma=10 \mathrm{MPa}$. Four different displacement proportional loading paths are performed with Quadri relation, with $k \neq 0$ around $\tan \left(15^{\circ}\right)$. Increment $d \gamma$ is used as the second loading parameter. The resulting stress paths appear in Figure 17(a) : for all tests, the stress state reaches the plastic limit criterion, where it evolves before reaching a final point (both $\tau$ and $\sigma$ reach a constant value). As explained in details in a previous work [13], these evolutions and the final state depend on the imposed dilation 


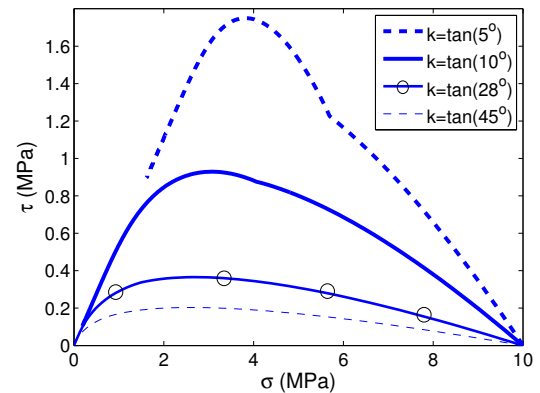

(a) $\tau(\sigma)$

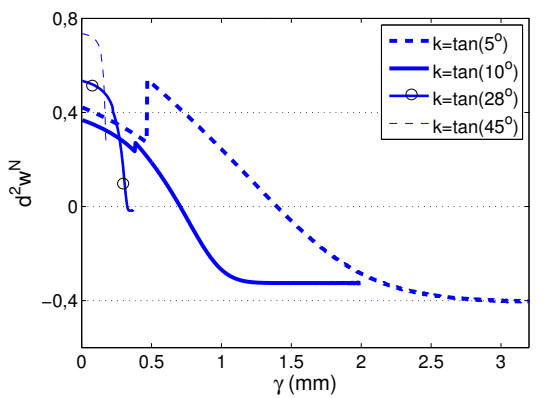

(c) $d^{2} w^{N}(\gamma)$

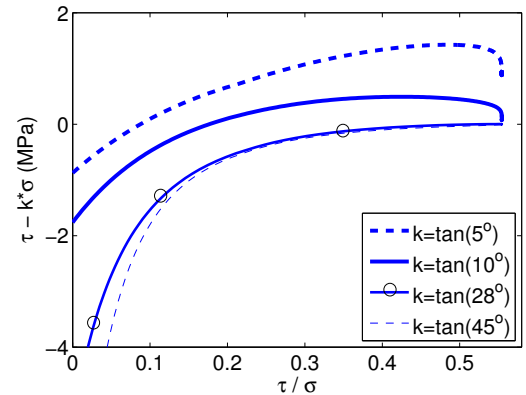

(b) $\tau-k \sigma=f(\tau / \sigma)$

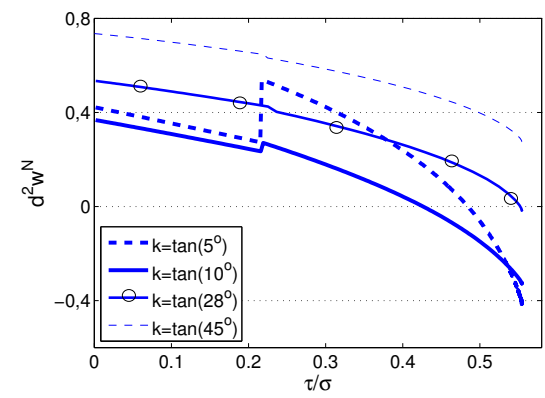

(d) $d^{2} w^{N}(\tau / \sigma)$

FiguRE 17 - Different displacement proportional loading paths 
rate (through parameter $k$ ) compared to the material dilation rate of the joint on the plastic limit criterion given by its flow rule (which was presented in Figure 14 for this set of parameters). For $k=\tan \left(5^{\circ}\right)$ for example, the stress state evolves along the plastic limit criterion until a $\sigma$-value such that $\psi(\sigma)=5^{\circ}$ : $\sigma \approx 2 \mathrm{MPa}$, which corresponds to the results of Figure 14 .

Values of $\tau-k \sigma$, that have to pass through a maximum in order to get instability, are monitored and plotted in Figure 17(b) according to the ratio $\tau / \sigma$. Note that this ratio increases monotonously for all tests, until maximum value of $\tan (\varphi)$

Evolutions of second order work are finally considered, according to $\gamma$ (Figure $17(\mathrm{c}))$ or ratio $\tau / \sigma$ (Figure $17(\mathrm{~d})$ ). Consideration of ratio $\tau / \sigma$ makes the curve easier to analyze. As in Figure 16(b), some sharp variations can be observed around $\tau / \sigma=\tan \left(\varphi_{c}\right)$, for loadings close to the CND one ( $k$ close to 0$)$. This being excepted, all curves decrease according to $\tau / \sigma$. Negative values are reached for $k \in\left[\tan \left(5^{\circ}\right) ; \tan \left(28^{\circ}\right)\right]$. Instability is obtained for lowest shearing (lowest ratio $\tau / \sigma)$ for $k=\tan \left(10^{\circ}\right)$. Indeed Figure 13 showed that the instability cones open around this value. Loading path $k=\tan \left(28^{\circ}\right)$ is included within the cones where their openings are maximal : instability is obtained just before the plastic limit criterion. Whereas, for $k=\tan \left(45^{\circ}\right)$, negative values of $d^{2} w^{N}$ cannot be reached. For all unstable cases, vanishing of $d^{2} w^{N}$ corresponds exactly, as it was theoretically expected, to maximum values of $\tau-k \sigma$ (see Figure 17(b) compared with $17(\mathrm{~d}))$.

No failure occurs during these numerical tests since they are fully displacement controlled : with $d u+k d \gamma=0$ and $d \gamma=c s t$. This instability (negative values of $d^{2} w$ ) triggers failure for mixed loading parameters. Figure 17 shows clearly that those failures would occur before plastic limit criterion : peaks of $\tau-k \sigma$ occur before maximum value of $\tau / \sigma$.

\section{$4 \tau$ - constant loading paths}

For rock joints stress proportional loading paths are governed by :

$$
\left\{\begin{array}{c}
d \tau-k d \sigma=0 \\
d \sigma=c s t \text { or } d u+k d \gamma=c s t
\end{array}\right.
$$

Using Equation (21), as in section 3.2, we show that, along such paths :

$d^{2} w=0 \Leftrightarrow d(u+k \gamma)=0$ if $\sigma$ is imposed, or $d \sigma=0$ if $u+k \gamma$ is imposed

Depending on the control mode, instability is obtained for decreases, after reaching a maximum, either of $u+k \gamma$ in case of full stress-controls $(d(\tau-k \sigma)$ and $d \sigma$ imposed $)$, or decreases of $\sigma$ in case of mixed $\operatorname{control}(d(\tau-k \sigma)$ and $d(u+k \gamma)$ imposed).

We focus now to the case $k=0$, that is $\tau$-constant loading paths. 


\subsection{Theoretical discussion}

This loading path corresponds to the case of Equation (28), for a vanishing $k$. It would appear for example for water-saturated rock joints, in which changes in the water pressure modifies the value of $\sigma$, but not $\tau$.

In three dimensions, monitored increases in water pore pressure lead to $q$ constant drained paths. There are some experimental evidences of diffuse failure before Mohr-Coulomb criterion along such paths, for loose sands [16, 17, 54]. Theoretically, this type of paths were discussed in [14] for example, using the second order work criterion. This section presents what happens when rock joints are considered.

Under the constraint of $d \tau=0$, we have :

$$
d^{2} w=d \sigma d u+d \tau d \gamma=d \sigma d u=\left(N_{\gamma} d \gamma+N_{u} d u\right) d u
$$

If the case $d u=0$ (corresponding to a CND loading and handled in section 3.1.1) is excluded we can write :

$$
d^{2} w=d u^{2}\left(N_{u}+N_{\gamma} \frac{d \gamma}{d u}\right)
$$

Since $d \tau=G_{\gamma} d \gamma+G_{u} d u=0$, the ratio $d \gamma / d u$ is determined, and :

$$
d^{2} w=d u^{2}\left(N_{u}-N_{\gamma} \frac{G_{u}}{G_{\gamma}}\right)=\frac{d u^{2}}{G_{\gamma}}\left(N_{u} G_{\gamma}-N_{\gamma} G_{u}\right)
$$

The determinant of the constitutive matrix appears : $N_{u} G_{\gamma}-N_{\gamma} G_{u}=\operatorname{det}(M)$; finally :

$$
d^{2} w=\frac{d u^{2}}{G_{\gamma}} \operatorname{det}(M)
$$

As for CND paths in section 3.1.1, instability depends on the link between deviatoric parts of stresses and strains : the sign of $G_{\gamma}$. For rock joints along $\tau$ constant paths, if $G_{\gamma}>0$ failures are only possible on the plasticity limit criterion :

$$
\text { For } G_{\gamma}>0, d^{2} w=0 \text { while } \tau=c s t \Leftrightarrow \operatorname{det}(M)=0
$$

\subsection{Numerical results}

Now that $\tau$ constant paths have been discussed analytically for the Quadri case, we consider them numerically for the INL2 case. Six $\tau$ constant paths are simulated with the INL2 relation (Equation (20)). Three different initial states are considered, all with the same value of $\sigma: \sigma=10 \mathrm{MPa}$, but with three different values of $\tau: \tau \in\left[0.3 \tau_{\max } ; 0.7 \tau_{\max } ; 0.9 \tau_{\max }\right]$, with $\tau_{\max }=\sigma \tan (\varphi)$. For $\sigma=10 \mathrm{MPa}, \tau$-values greater than $0.6 \tau_{\max }$ should correspond to the bifurcation domain of the rock joint constitutive relation (see Figure 9).

For each stress state, $\tau$ constant paths are performed for $d \sigma=c s t>0$ and $d \sigma=-c s t<0$. Value $c s t=|d \sigma|=10 \mathrm{kPa}$ was used, which is small enough to 
have no influence on the results. Such paths being completely stress-controlled, Equation (20) has to be inverted in order to determine $\overrightarrow{d l}$ which corresponds to the imposed stress variation $\overrightarrow{d \sigma}=(d \tau=0, d \sigma=+/-c s t)$. This inversion becomes impossible when the plastic limit criterion is reached for $d \sigma<0$. On the plastic limit criterion, there is no $\overrightarrow{d l}$ leading to $(d \tau=0, d \sigma<0)$ : otherwise, the further stress state would not obey the limit criterion. We have shown in [13] that the INL2 constitutive relation obeys to a Mohr-Coulomb type plastic limit criterion, close to the one of the Quadri relation, but with a slightly greater friction angle value : $\varphi_{I N L 2}=30.5^{\circ} \pm 0.5^{\circ}\left(\approx \varphi=29^{\circ}\right)$.

This plastic limit criterion appears in Figure 18(a), where the imposed stress paths are plotted. They all lie below the straight line $\tau=\sigma \tan \left(\varphi_{I N L 2}\right)$.

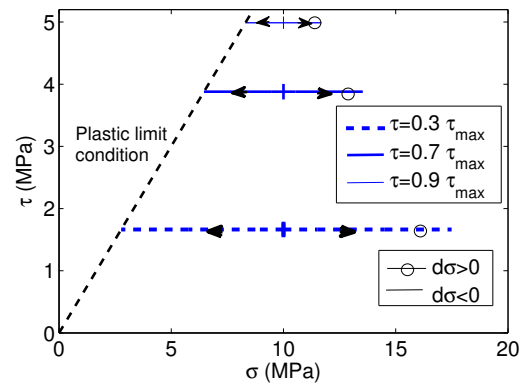

(a) Stress controlled paths

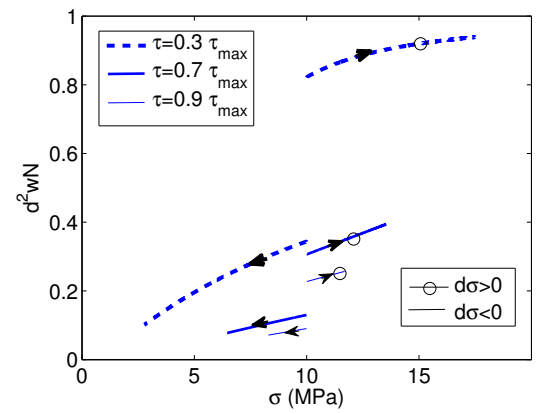

(c) $d^{2} w^{N}(\sigma)$

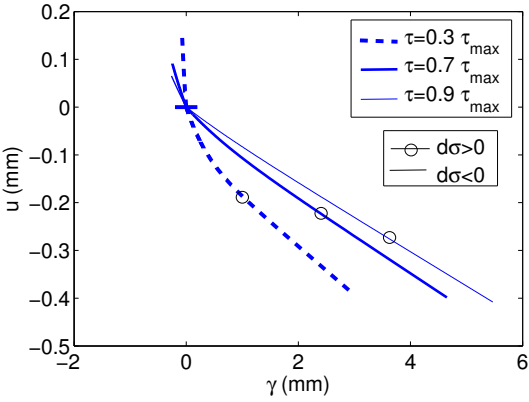

(b) Displacement responses

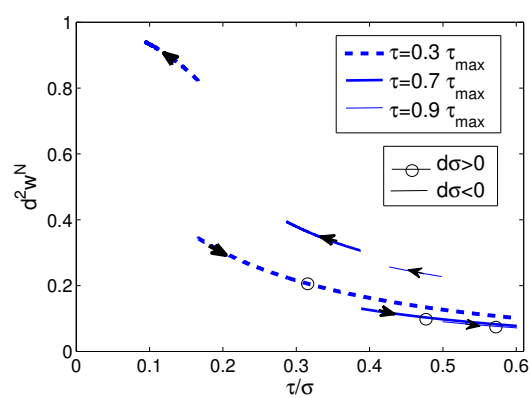

(d) $d^{2} w^{N}(\tau / \sigma)$

FIgURE 18 - Behaviour of the joint along $\tau$ constant paths

In Figure 18(b) the resulting displacements are presented : dilatancy $(d u<$ 0 ) occurs for imposed $d \sigma<0$, and values of relative displacements are greater for unloading mode $(d \sigma<0)$, and as the plastic limit criterion is close (for greater $\tau$ values, for example).

According to Figure 18(c) we verify that no negative or nil value of $d^{2} w^{N}$ 
is obtained through these paths with the INL2 relation, as it was deduced from the analytical considerations of paragraph 4.1. Since these paths never go along the plasticity limit criterion (following a $\tau$ constant path on the plastic limit criterion would require $d \sigma=d \tau=0$ ), no nil value is also obtained on this criterion.

Figure 18(d) also illustrates that the (normalised) second order work decreases again with respect to $\tau / \sigma$ ratio.

\section{$5 \quad$ Failure rules}

Failures occuring on plastic limit criterion do not occur with any mechanism. Indeed the direction of the resulting displacements is determined (unlike their values) because of the existence of the flow rule. Darve et al. (Equation (28) of [14]) derived similar failure rules for soils under axisymmetric conditions, when the second order work is vanishing.

For failures before plastic limit criterion, these authors showed that links between increments of stresses and strains $\left(d \varepsilon_{1}\right.$ and $d \sigma_{3}$ in [14]) still exist, as for the different components of displacements (or strains) during failures on plastic limit criterion. Such failure rules for rock joints is here derived.

As in [14], the starting point is to write the constitutive relation, paying attention to the relevant loading variables : $d u+k d \gamma$ and $d \tau-k d \sigma$, considering the framework of sections 3 or 4 . This is always possible, since $(d \tau-k d \sigma, d \gamma)$ and $(d u+k d \gamma, d \sigma)$ are conjuguated variables with respect to energy : $(\tau-k \sigma) \gamma+$ $(u+k \gamma) \sigma=\tau \gamma+\sigma u$. For piecewise linear relations (6), we rewrite them, after few calculations, under following form :

$$
\left(\begin{array}{c}
d \tau-k d \sigma \\
d u+k d \gamma
\end{array}\right)=\left(\begin{array}{cc}
\frac{\operatorname{det}(M)}{N_{u}} & \frac{G_{u}}{N_{u}}-k \\
k-\frac{N_{\gamma}}{N_{u}} & \frac{1}{N_{u}}
\end{array}\right)\left(\begin{array}{c}
d \gamma \\
d \sigma
\end{array}\right)=M^{\prime}\left(\begin{array}{c}
d \gamma \\
d \sigma
\end{array}\right)
$$

Under either stress or displacement proportionnal loading conditions, one of the two terms of the vector $(d u+k d \sigma, d \tau-k d \sigma)$ vanishes. Failure corresponds to a cancellation of the second term, while $(d \gamma, d \sigma) \neq \overrightarrow{0}$.

This first implies a bifurcation criterion [14] :

$$
\operatorname{det}\left(M^{\prime}\right)=0
$$

Which is linked to cancellation of second order work. With $P$ the polynom of Equation (9), we get :

$$
\operatorname{det}\left(M^{\prime}\right)=\frac{P(-k)}{N_{u}}=\frac{P(d u / d \gamma)}{N_{u}}=\frac{d^{2} w(\overrightarrow{d l})}{N_{u}}
$$


Then, we can now derive the failure rule using the set of solutions $(d \gamma, d \sigma)$ which now exist :

$$
\left(k-\frac{N_{\gamma}}{N_{u}}\right) d \gamma+\frac{d \sigma}{N_{u}}=0
$$

As explained in [14], from the generalized limit stress state : $(d \tau-k d \sigma=$ $0, d u+k d \gamma=0)$, response increments are unknow : an infinity of solutions exist, but they all obey to Equation (38). Note that $k$ appears in Equation (38), as $R$ in Equation (28) of [14].

\section{Conclusion}

In this paper we have studied the mechanical stability of rock joints, restricting to the case of positive normal stiffnesses. The second order work criterion, already used for soils, was applied to rock joints which are seen as interface media. Related to this stability criterion, the bifurcation domain and directions of instability for a given rock joint were determined. As for soils, in case of nonassociativeness, failure before the plastic limit criterion can occur, for some loading directions forming cones of instability. Coupling features of the joint (e.g., dilatancy) appear to have a key role for the stability of the joint. Indeed, instability is obtained depending on the magnitude of the corresponding moduli, compared between each other, and with the diagonal rigidities. The instability patterns are also different depending on this moduli comparison ( $d \sigma$ with same sign than $d \gamma$, or not).

We applied the theoretical study to two given rock joint constitutive relations : the Quadri and INL2 ones [13]. The bifurcation domains and instability cones were illustrated in these cases, with some sensitivity analyses. With these relations (after having modified some values of parameters), attention was particularly paid to two loading paths : constant normal displacement $(d u=0)$, or constant tangential stress $(d \tau=0)$. No failure was obtained here before plastic limit criterion, but this would occur along these two paths if the deviatoric stress $\tau$ decreases with the deviatoric "strain" $\gamma$ along undrained paths, exactly as found for loose soils. We also illustrated that such failures happen for loading directions belonging to instability cones (which implies that the mechanical state belongs to the bifurcation domain), and if appropriate loading parameters are used (necessarily mixed ones). The failure rules were determined : they give the failure mechanisms if such failures occur. Furthermore we showed that the second order work seems to decrease during shearing, irrespective of the loading direction. This means, roughly speaking, that shearing leads to instability. However, the instability becomes a failure or does not, depending on the loading directions and on the loading parameters .

Regarding rock slope stability, which is the framework of this study, an analysis of an existing cliff was presented in another work [55]. From a numerical model of the cliff, using the INL2 relation, conditions of instability were obtained in some simulations, even if they did not trigger failure. A global value of the 
second order work (only local values were considered) needs to be used to deal with such boundary problems.

These results can be applied to any interface media, that is soil-structure interfaces (e.g., around piles), but also natural interfaces : for example rock faults responsible for earthquakes.

\section{APPENDIX}

We derived in section 1.2 the links existing between the orientation of the cones of instability and the signs of $d \gamma$ and $G_{u}-N_{\gamma}$. The orientation of the cones are determined through the signs of $d \sigma_{k}{ }^{u}, k=1 ; 2$, defined in Figure 1. We have, due to equations (6) and (11) :

$$
\begin{aligned}
d \sigma_{k}^{u} & =N_{\gamma} d \gamma+N_{u} d u=d \gamma\left(N_{\gamma}+N_{u} \frac{-\left(G_{u}+N_{\gamma}\right)+f_{k} \sqrt{\Delta}}{2 N_{u}}\right) \\
& =\frac{d \gamma}{2}\left(N_{\gamma}-G_{u}+f_{k} \sqrt{\Delta}\right)=\frac{d \gamma}{2} L_{k}
\end{aligned}
$$

We have in Equation (39) $f_{k}=1$ for one branch of the cone and $f_{k}=-1$ for the other. The sign of $d \sigma$ depends partly on sign of $L_{k}=N_{\gamma}-G_{u}+f_{k} \sqrt{\Delta}$. And the sign of $L_{k}$ depends itself on the one of $N_{\gamma}-G_{u}$.

If $N_{\gamma}-G_{u}>0$ we get obviously $L_{k}>0$ for branch $k$ such as $f_{k}=1$. For the other branch in this case :

$$
\begin{aligned}
L_{k} \geqslant 0 & \Leftrightarrow N_{\gamma}-G_{u} \geqslant \sqrt{\Delta}>0 \\
& \Leftrightarrow\left(N_{\gamma}-G_{u}\right)^{2} \geqslant \Delta \\
& \Leftrightarrow\left(N_{\gamma}-G_{u}\right)^{2} \geqslant\left(N_{\gamma}+G_{u}\right)^{2}-4 N_{u} G_{\gamma} \\
& \Leftrightarrow N_{u} G_{\gamma}-N_{\gamma} G_{u} \geqslant 0 \Leftrightarrow \operatorname{det}(M) \geqslant 0
\end{aligned}
$$

Determinant of the constitutive matrix appears. It is physically positive (vanishing only on plastic limit criterion), which proves that $L_{k}$ is also positive for both branchs of the cone : both $d \sigma_{k}^{u}$ have then the same sign as $d \gamma$, for $N_{\gamma}-G_{u}>0$.

If $N_{\gamma}-G_{u}<0$. For branch $k$ with $f_{k}=-1$, we get easily again $L_{k}<0$. For other branch with $f_{k}=1$, we show that $L_{k}$ is also negative because $\operatorname{det}(M)$ is positive :

$$
\begin{aligned}
\operatorname{det}(M) \geqslant 0 & \Leftrightarrow N_{u} G_{\gamma}-N_{\gamma} G_{u} \geqslant 0 \\
& \Leftrightarrow \frac{1}{4}\left(4 N_{u} G_{\gamma}+\left(G_{u}-N_{\gamma}\right)^{2}-\left(G_{u}+N_{\gamma}\right)^{2}\right) \geqslant 0 \\
& \Leftrightarrow-\Delta+\left(G_{u}-N_{\gamma}\right)^{2} \geqslant 0 \\
& \Leftrightarrow \sqrt{\Delta} \leqslant G_{u}-N_{\gamma}: \text { both terms, } \Delta \text { and } G_{u}-N_{\gamma}, \text { are } \geqslant 0 \\
& \Leftrightarrow L_{k} \leqslant 0
\end{aligned}
$$

In this case $N_{\gamma}-G_{u}<0$, signs of both $d \sigma^{u}{ }_{k}$ are opposite to the one of $d \gamma$. We end up with the conditions presented in Table 1. 


\section{Références}

[1] Bandis SC, Lumsden AC, Barton NR. Fundamentals of rock joint deformation. Int. J. Rock Mech. Min. Sci. \& Geomech. Abstr. 1983; 20(6) :249-268.

[2] Saeb S, Amadei B. Modelling rock joints under shear and normal loading. Int. J. Rock Mech. Min. Sci. \& Geomech. Abstr. 1992; 29(3) :267-278.

[3] Pereira JP. Rolling friction and shear behaviour of rock discontinuities filled with sand. Int. J. Rock Mech. \& Min. Sci. 1997; 34 :244e1-244e17.

[4] Boulon M, Armand G, Hoteit N, Divoux P. Experimental investigations and modelling of shearing of calcite healed discontinuities of granodiorite under typical stresses. Engineering Geology 2002; 64 :117-133.

[5] Drucker D. Limit analysis of two and three dimensional soil mechanics problems. Journal of the Mechanics and Physics of Solids 1953; 1(4) :217226.

[6] Livesley R. Limit analysis of structures formed from rigid blocks. Int. J. Numer. Method. Eng. 1978; 12 :1853-1871.

[7] Aydan O, Kawamoto T. Stability of slopes and underground openings against flexural toppling and their stabilisation. Rock Mech. Rock Engng. $1992 ; \mathbf{2 5}(3): 143-165$.

[8] Durand A, Vargas-Jr E, Vaz L. Applications of numerical limit analysis (nla) to stability problems of rock and soil masses. International Journal of Rock Mechanics 83 Mining Sciences 2006; 43 :408-425.

[9] Adhikary DP, Dyskin AV. Modelling of progressive and instantaneous failures of foliated rock slopes. Rock Mech. Rock Engng. 2007 ; 40(4) :349-362.

[10] Duriez J, Darve F, Donzé FV. A discrete modeling-based constitutive relation for infilled rock joints. International Journal of Rock Mechanics 8 Mining Sciences 2011; 48(3) :458-468, doi :10.1016/j.ijrmms.2010.09.008.

[11] Darve F. The expression of rheological laws in incremental form and the main classes of constitutive equations. Geomaterials Constitutive Equations and Modelling, Darve F (ed.). Elsevier Applied Science : London, 1990; $123-148$.

[12] Plesha ME. Constitutive models for rock discontinuities with dilatancy and surface degradation. International Journal for Numerical and Analytical Methods in Geomechanics 1987; 11 :345-362.

[13] Duriez J, Darve F, Donzé FV. Incrementally non-linear plasticity applied to rock joint modelling. International Journal for Numerical and Analytical Methods in Geomechanics 2013; 37(5) :453-477, doi :10.1002/nag.1105.

[14] Darve F, Servant G, Laouafa F, Khoa H. Failure in geomaterials : continuous and discrete analyses. Comput. Methods Appl. Mech. Engrg. 2004; $193: 3057-3085$.

[15] Khoa H, Georgopoulos I, Darve F, Laouafa F. Diffuse failure in geomaterials : Experiments and modelling. Computers and Geotechnics 2006; $33: 1-14$. 
[16] di Prisco C, Imposimato S. Experimental analysis and theoretical interpretation of triaxial load controlled loose sand specimen collapses. Mechanics of Cohesive-Frictional Materials 1997; 2 :93-120.

[17] Chu J, Leroueil S, Leong W. Unstable behaviour of sand and its implication for slope instability. Canadian Geotechnical Journal 2003; 40 :873-885.

[18] Vardoulakis I, Sulem J. Bifurcation analysis in geomechanics. Blackie Academic \& Professional : London, 1995.

[19] Desrues J, Chambon R. Shear band analysis and shear moduli calibration. International Journal of Solids and Structures 2002 ; 39(13-14):3757-3776.

[20] Desrues J, Viggiani G. Strain localization in sand : an overview of the experimental results obtained in grenoble using stereophotogrammetry. International Journal for Numerical and Analytical Methods in Geomechanics 2004 ; 28(4) :279-321, doi :10.1002/nag.338.

[21] Rudnicki J, Rice JR. Conditions for the localisation of deformation in pressure sensitive dilatant materials. J. Mech. Phys. Solids 1975 ; 23 :371-394.

[22] Rice JR. The localization of plastic deformation. Theoretical and Applied Mechanics, Koiter WT (ed.), North-Holland Publishing Company, 1976.

[23] Laouafa F, Darve F. Modelling of slope failure by a material instability mechanism. Computers and Geotechnics $2002 ; 29$ :301-325.

[24] Darve F, Sibille L, Daouadji A, Nicot F. Bifurcations in granular media : macro- and micro-mechanics approaches. C. R. Mécanique 2007 ; 335 :496515.

[25] Nicot F, Darve F. A micro-mechanical investigation of bifurcation in granular materials. International Journal of Solids and Structures 2007; 44 :6630-6652.

[26] Sibille L, Donzé FV, Nicot F, Chareyre B, Darve F. From bifurcation to failure in a granular material : a dem analysis. Acta Geotechnica 2008; 3(1) :15-24.

[27] Lyapunov A. Problème général de la stabilité des mouvements. Annales de la faculté des sciences de Toulouse 1907; 9 :203-274.

[28] Hill R. A general theory of uniqueness and stability in elastic-plastic solids. J. of the Mech. and Phys. of Solids 1958; 6 :239-249.

[29] Darve F, Chau B. Constitutive instabilities in incrementally non-linear modelling. Constitutive Laws for Engineering Materials. Theory and Applications, Desai C, Krempl E, Kiousis P, Kundu T (eds.). Elsevier, 1987 ; 301-310.

[30] Daouadji A, Darve F, Gali HA, Hicher PY, Laouafa F, Lignon S, Nicot F, Nova R, Pinheiro M, Prunier F, et al.. Diffuse failure in geomaterials : Experiments, theory and modelling. International Journal for Numerical and Analytical Methods in Geomechanics 2011; 35(16) :1731-1773, doi : $10.1002 /$ nag.975. 
[31] Daouadji A, AlGali H, Darve F, Zeghloul A. Instability in granular materials : Experimental evidence of diffuse mode of failure for loose sands. Journal of Engineering Mechanics 2010; 136(5) :575-588, doi : 10.1061/_ASCE \_EM.1943-7889.101.

[32] Daouadji A. Errata for instability in granular materials : Experimental evidence of diffuse mode of failure for loose sands. Journal of Engineering Mechanics 2010; 136(8) :1065.

[33] Bigoni D, Hueckel T. Uniqueness and localization i. associative and nonassociative elastoplasticity. International Journal of Solids and Structures 1991; 28(2) :197-213.

[34] Nicot F, Darve F. Diffuse and localized failure modes : Two competing mechanisms. International Journal for Numerical and Analytical Methods in Geomechanics 2011; 35(5) :586-601, doi :10.1002/nag.912.

[35] Challamel N, Nicot F, Lerbet J, Darve F. On the stability of nonconservative elastic systems under mixed perturbations. European Journal of Environmental and Civil Engineering 2009; 13(3) :347-367.

[36] Nicot F, Sibille L, Darve F. Bifurcation in granular materials : An attempt for a unified framework. International Journal of Solids and Structures 2009 ; 46(22-23) :3938 - 3947, doi :10.1016/j.ijsolstr.2009.07.008.

[37] Nicot F, Daouadji A, Laouafa F, Darve F. Second-order work, kinetic energy and diffuse failure in granular materials. Granular Matter 2011; 13 :19-28, doi :10.1007/s10035-010-0219-2.

[38] Nicot F, Sibille L, Darve F. Failure in rate-independent granular materials as a bifurcation toward a dynamic regime. International Journal of Plasticity 2012; 29 :136 - 154, doi :10.1016/j.ijplas.2011.08.002.

[39] Nova R. Liquefaction, stability, bifurcations of soil via strainhardening plasticity. Numerical Methods for Localisation and Bifurcation of Granular Bodies, $1989 ; 117-132$.

[40] Nova R. Controllability of the incremental response of soil specimens subjected to arbitrary loading programmes. Journal of the Mechanical Behavior of Materials 1994; 5 :193-202, doi :10.1515/JMBM.1994.5.2.193.

[41] Imposimato S, Nova R. An investigation on the uniqueness of the incremental response of elastoplastic models for virgin sand. Mechanics of Cohesivefrictional Materials 1998; 3(1):65-87.

[42] Darve F, Labanieh S. Incremental constitutive law for sands and clays. simulations of monotonic and cyclic tests. International Journal for Numerical and Analytical Methods in Geomechanics 1982; 6 :243-275.

[43] Darve F, Flavigny E, Meghachou M. Yield surfaces and principle of superposition : Revisit through incrementally non-linear constitutive relations. International Journal of Plasticity 1995; 11(8) :927-948, doi : 10.1016/S0749-6419(95)00037-2. 
[44] Mollema P, Antonellini M. Compaction bands : a structural analog for antimode i cracks in aeolian sandstone. Tectonophysics $1996 ; \mathbf{2 6 7}(1-4): 209$ 228, doi :10.1016/S0040-1951(96)00098-4.

[45] Olsson WA. Theoretical and experimental investigation of compaction bands in porous rock. J. Geophys. Res. 1999; 104(B4) :7219-7228, doi : 10.1029/1998JB900120.

[46] Issen KA, Rudnicki JW. Conditions for compaction bands in porous rock. $J$. Geophys. Res. 2000 ; 105(B9) :21 529-21 536, doi :10.1029/2000JB900185.

[47] Sibille L, Nicot F, Donzé FV, Darve F. Material instability in granular assemblies from fundamentally different models. International Journal for Numerical and Analytical Methods in Geomechanics 2007; 31 :457-481.

[48] Prunier F, Nicot F, Darve F, Laouafa F, Lignon S. Three-dimensional multiscale bifurcation analysis of granular media. Journal of Engineering Mechanics 2009; 135(6) :493-509.

[49] Nicot F, Challamel N, Lerbet J, Prunier F, Darve F. Bifurcation and generalized mixed loading conditions in geomaterials. International Journal for Numerical and Analytical Methods in Geomechanics 2011; 35(13) :14091431, doi :10.1002/nag.959.

[50] Nicot F, Challamel N, Lerbet J, Prunier F, Darve F. Some insights into structure instability and the second-order work criterion. International Journal of Solids and Structures 2012; 49(1) :132 - 142, doi : 10.1016/j.ijsolstr.2011.09.017.

[51] Darve F, Laouafa F. Instabilities in granular materials and application to landslides. Mech. Cohes. Fric. Mater. 2000 ; 5 :627-652.

[52] Nicot F, Darve F. Micro-mechanical investigation of material instability in granular assemblies. International Journal of Solids and Structures 2006 ; $43: 3569-3595$.

[53] Darve F, Nicot F. On flow rule in granular media : phenomenological and multi-scale views (part ii). International Journal for Numerical and Analytical Methods in Geomechanics 2005; 29 :1411-1432.

[54] Daouadji A, Darve F, Gali HA, Lejeune A, Jrad M. Experimental and numerical analyses of failure in very loose sands. European Journal of Environmental and Civil Engineering 2009; 13(2):149-165.

[55] Merrien-Soukatchoff V, Duriez J, Gasc M, Darve F, Donzé FV. Mechanical stability analyses of fractured rock slopes. Rockfall Engineering, Lambert S, Nicot F (eds.). John Wiley \& Sons, New York, ISTE ltd, London, 2011. 